\title{
Synthesis of 1,3-Diynes via Detelluration of Bis(ethynyl)tellurides
}

\author{
Hélio A. Stefani, ${ }^{* a}$ Jesus M. Pena, ${ }^{a}$ Julio Zukerman-Schpector ${ }^{b}$ and \\ Edward R. T. Tiekink ${ }^{c}$ \\ ${ }^{a}$ Faculdade de Ciências Farmacêuticas, Universidade de São Paulo, São Paulo-SP, Brazil \\ ${ }^{b}$ Departamento de Química, Universidade Federal de São Carlos, 13565-905 São Carlos-SP, Brazil \\ 'Department of Chemistry, University of Malaya, Kuala Lumpur 50603, Malaysia
}

\begin{abstract}
Neste artigo é descrita a síntese de sistemas diínicos conjugados contendo substituintes elétronatratores e elétron-doadores via a deteluração catalizada por paládio de bis-(ariletinil)teluretos e bis-(alquiletinil)teluretos. Este procedimento foi realizado sob condições atmosféricas em DMF usando $\mathrm{Pd}(\mathrm{Oac})_{2}$ como catalisador e $\mathrm{AgOAc}$ como um aditivo na presença de trietilamina. Esta rota oferece acesso eficiente a sistemas diínicos conjugados em um curto período de tempo. A estrutura cristalográfica por difração de raios X do telureto de bis(p-toluiletinila) e a conformação no estado sólido mostram uma cadeia supramolecular alinhada ao longo do eixo b, sustentada por interações $\mathrm{CH} . . . \pi$.
\end{abstract}

The synthesis of symmetric conjugated diyne systems with electron-withdrawing or electrondonating substituents via a palladium-catalyzed detelluration of bis(arylethynyl)tellurides and bis(alkylethynyl)tellurides is described. This procedure is effected under atmospheric conditions in DMF using $\mathrm{Pd}(\mathrm{OAc})_{2}$ as a catalyst and $\mathrm{AgOAc}$ as an additive in the presence of triethylamine. This route offers efficient access to conjugated diyne systems in short reaction time. X-ray crystallographic structure and solid-state conformation of bis(p-tolylethynyl)telluride show a supramolecular chain aligned along the $b$ axis, sustained by C-H... $\pi$ interactions.

Keywords: tellurium, bis(phenylethynyl)tellurides, bis(alkynylethynyl)tellurides, detelluration, diynes, palladium

\section{Introduction}

Organotellurium compounds play an important role in organic synthesis, and they have received considerable attention because of their potential availability and useful biological activity. ${ }^{1}$ As reported by Bergman and co-workers ${ }^{2}$ more than three decades ago, aryltelluriums undergo detelluration upon treatment with degassed Raney nickel to afford biaryl compounds. Although the reaction is interesting and synthetically useful, the necessity of more than a stoichiometric amount of the required metal is still a serious drawback. However, attempted transition metalcatalyzed detellurations have been unsuccessful to date. ${ }^{2,3}$

Compounds containing chains of conjugated triple bonds $s^{4,5}$ are of paramount importance as versatile and useful building blocks in organic synthesis. Among these compounds, 1,3-butadiynes ${ }^{6}$ have been prominently

*e-mail: hstefani@usp.br utilized as substructures in the formation of valuable intermediates for natural products ${ }^{7}$ and pharmaceuticals such as antitumor, ${ }^{8}$ antibacterial,${ }^{9}$ anti-inflammatory ${ }^{10}$ and antifungal agents. ${ }^{11}$

These conjugated diynes also serve as the core functional group in organic molecular materials such as linearly $\sigma$-conjugated acetylenic oligomers and polymers, ${ }^{12}$ macrocycles ${ }^{13}$ (Figure 1), and supramolecular scaffolds. ${ }^{14}$ Oxidative dimerization of $s p$-hybridized terminal alkynes mediated by $\mathrm{Cu}(\mathrm{I})$ or $\mathrm{Cu}(\mathrm{II})$ salts under either catalytic or stoichiometric conditions is the most commonly used synthetic methodology for obtaining symmetrically substituted 1,3-butadiyne.

These approaches include Glaser's coupling, ${ }^{15}$ Eglinton's coupling, ${ }^{16}$ and Hay's coupling. ${ }^{17}$ In these reactions, the transmetalation of an alkynyl group to copper is proposed to generate an alkynylcopper species that undergoes subsequent oxidative dimerization to give the corresponding 1,3-butadiynes. ${ }^{18}$ 


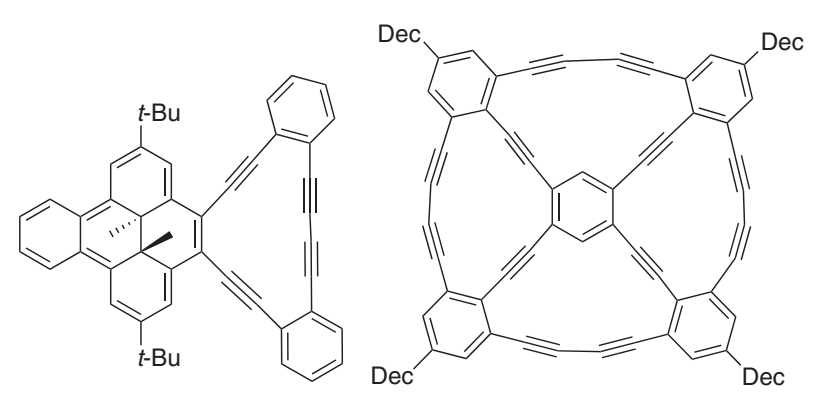

Figure 1. Structure of diyne macrocycles.

\section{Results and Discussion}

Herein, we describe a convenient protocol for the synthesis of symmetrical conjugated diynes through the palladium-catalyzed detelluration of functionalized bis(arylethynyl)tellurides and bis(alkylethynyl) tellurides at room temperature in presence of air (Scheme 1).

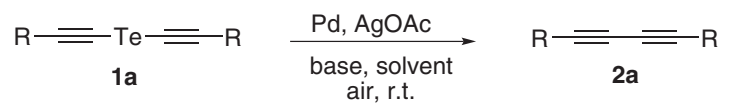

Scheme 1. Synthesis of conjugated diynes.

The approach to preparing symmetrical conjugated diynes $\mathbf{2 a - j}$ was based on a palladium-catalyzed detelluration reaction of functionalized bis(phenylethynyl)tellurides and bis(alkylethynyl) tellurides 1a-j. The parent precursors bis(arylethynyl)telluride 1a-j were conveniently prepared in good to moderate yields according to the procedure described by Engman and Stern. ${ }^{19}$
We initially optimized the conditions for the detelluration of functionalized bis(phenylethynyl)telluride $\mathbf{1}$. To find optimal conditions for the detelluration reaction, bis(phenylethynyl) telluride 1a was selected as a model substrate, and a variety of catalysts were screened as described in Table 1. All reactions were monitored by TLC and GC/MS.

We initially surveyed palladium catalysts for use in this detelluration reaction. A comparison of different palladium catalysts such as $\mathrm{PdCl}_{2}(\mathrm{dppf}) \cdot \mathrm{CH}_{2} \mathrm{Cl}_{2}, \mathrm{Pd}(\mathrm{OAc})_{2}$, $\mathrm{PdCl}_{2} \mathrm{PEPPSI}^{-} \mathrm{Pr}, \mathrm{PdCl}_{2}$, and $\mathrm{PdCl}_{2}\left(\mathrm{PhCN}_{2}(72 \%, 52 \%\right.$, $50 \%, 31 \%$ and $46 \%$ yields, respectively). Although the best yield (72\% yield) was obtained using $\mathrm{PdCl}_{2}$ (dppf). $\mathrm{CH}_{2} \mathrm{Cl}_{2}$, we choose $\mathrm{Pd}(\mathrm{OAc})_{2}$ as catalyst due its availability in our laboratory. No product formation was observed using catalysts such as $\mathrm{Fe}(\mathrm{acac})_{2}, \mathrm{CuCl}$ and $\mathrm{NiCl}_{2}$ (dppe) (entries $8-10)$. The reaction also did not proceed in the absence of a Pd catalyst (Table 1, entry 1).

One equiv. of $\mathrm{AgOAc}$ was used as an additive, along with 4 equiv. of triethylamine, and $\mathrm{MeOH}$ as solvent.

After the determination of the optimal catalyst for this transformation, we then studied the influence of the base. In our initial attempts, we used triethylamine, and the desired compound was obtained in $72 \%$ yield. We also attempted the same reaction with some other organic and inorganic bases such as $\mathrm{NaOAc}, \mathrm{Cs}_{2} \mathrm{CO}_{3}, \mathrm{~K}_{2} \mathrm{CO}_{3}$, DIPEA, pyridine and cyclohexylamine, obtaining the detelluration product in yields ranging from $27 \%$ to $67 \%$.

To further determine the optimal conditions for the detelluration reaction, we performed the model reaction in various solvents. When $\mathrm{MeOH}, 1,4$-dioxane, toluene and $\mathrm{CH}_{3} \mathrm{CN}$ were employed, the reaction yields were poor or moderate $(52 \%, 44 \%, 38 \%$ and $58 \%$, respectively). While

Table 1. Effect of a catalyst on the detelluration reaction of bis(phenylethynyl)telluride 1a

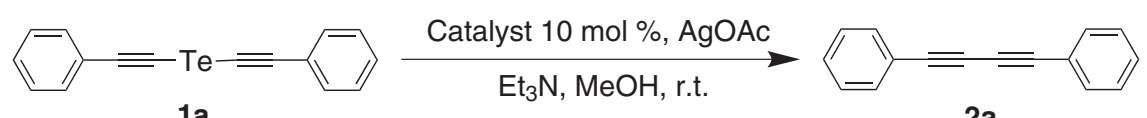

$1 a$

$2 a$

\begin{tabular}{|c|c|c|}
\hline entry & Catalyst $^{\mathrm{a}}$ & Yield / $(\%)^{\mathrm{b}}$ \\
\hline 1 & - & $\mathrm{nr}$ \\
\hline 2 & $\mathrm{PdCl}_{2}(\mathrm{dppf}) \cdot \mathrm{CH}_{2} \mathrm{Cl}_{2}$ & 72 \\
\hline 3 & $\mathrm{PdCl}_{2} \mathrm{PEPPSI}^{-} \mathrm{Pr}$ & 50 \\
\hline 4 & $\mathrm{Pd}(\mathrm{OAc})_{2}$ & 52 \\
\hline 5 & $\mathrm{PdCl}_{2}$ & 31 \\
\hline 6 & $\mathrm{PdCl}_{2}(\mathrm{PhCN})_{2}$ & 46 \\
\hline 7 & $\mathrm{Fe}(\mathrm{acac})_{2}$ & $\mathrm{nr}$ \\
\hline 8 & $\mathrm{CuCl}$ & $\mathrm{nr}$ \\
\hline 9 & $\mathrm{NiCl}_{2}(\mathrm{dppf})$ & $\mathrm{nr}$ \\
\hline
\end{tabular}

Reaction conditions: bis(phenylethynyl)telluride 1a (1 equiv.), catalyst (10 mol \%), $\mathrm{Et}_{3} \mathrm{~N}$ (4 equiv.), $\mathrm{AgOAc}\left(1.0\right.$ equiv.), $\mathrm{MeOH}(5 \mathrm{~mL})$, r.t. ${ }^{\mathrm{a}} 10 \mathrm{~mol} \%$ of

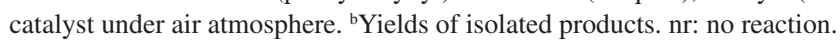


using DMSO as solvent provided a good yield (72\%), DMF provided the best yield of the desired product (86\%).

From these studies, it was determined that a reaction mixture containing 1.0 equiv. of bis(phenylethynyl)telluride 1a, 4 equiv. of $\mathrm{Et}_{3} \mathrm{~N}, 1$ equiv. of $\mathrm{AgOAc}$, and $10 \mathrm{~mol} \%$ of $\mathrm{Pd}(\mathrm{OAc})_{2}$ in $5 \mathrm{~mL}$ of $\mathrm{DMF}$ at room temperature stirred under atmospheric conditions for $60 \mathrm{~min}$ provided the best conditions for the synthesis of conjugated diyne 2a. To demonstrate the efficiency of this detelluration reaction, we then explored its generality with a variety of bis(arylethynyl)tellurides and bis(alkylethynyl)tellurides.

The results are summarized in Table 2 .
After optimizing the conditions for the synthesis of symmetrical conjugated diyne $\mathbf{2 a}$, it was synthesized a series of conjugated diynes (2a-j) in $21-86 \%$ yields (see Table 2 and Experimental). The reaction was carried out at room temperature. The reaction proceeded with electron-withdrawing substituents attached to the alkynyltelluride and with electron-donation substituents. All of the obtained products provided ${ }^{1} \mathrm{H}$ and ${ }^{13} \mathrm{C}$ NMR spectra that were in full agreement with their assigned structures.

On the basis of available literature ${ }^{20}$ we propose a possible catalytic cycle for the detelluration reaction of

Table 2. Detelluration reaction of bis(arylethynyl)tellurides and bis(alkylethynyl)tellurides
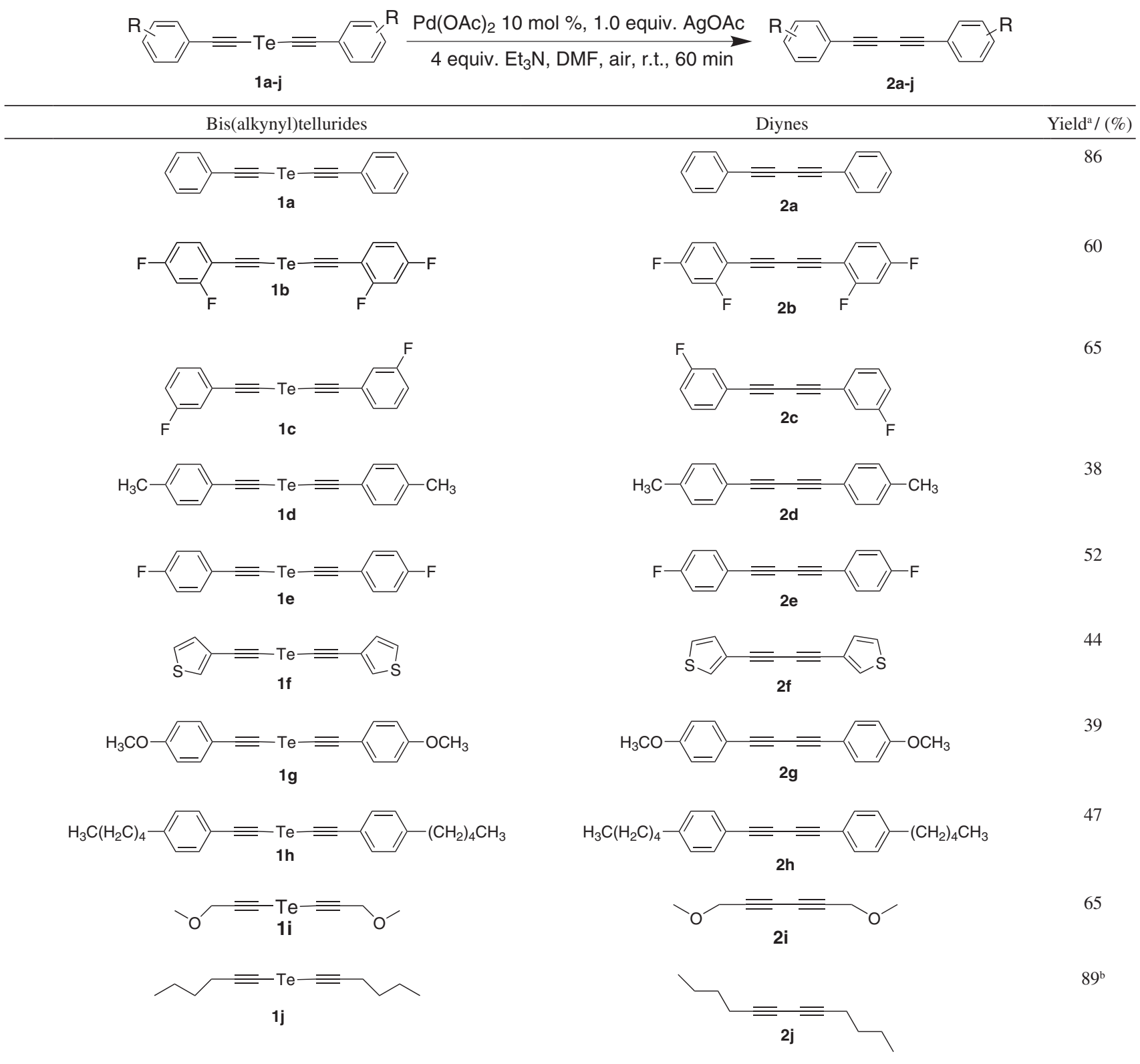

${ }^{\mathrm{a}}$ Isolated yields. ${ }^{\mathrm{b}}$ Yield by CG-MS, the product was not isolated by column. 


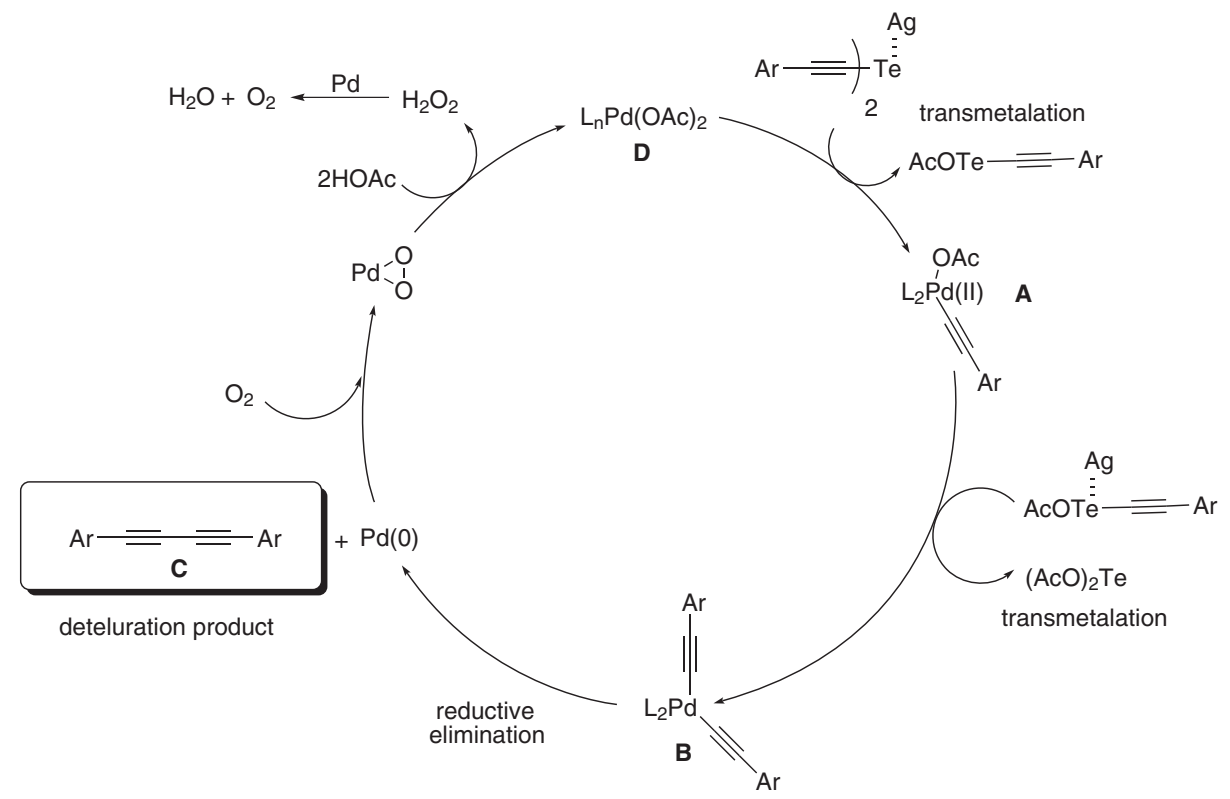

Figure 2. Possible catalytic cycle of the homocoupling reaction of alkynylditellurides.

bis(arylethynyl)tellurides and bis(alkylethynyl)tellurides as described in Figure 2.

According to this cycle the reaction proceeds by the formation of Pd(II) complex with acetylene followed by the conversion of this intermediate into another palladium species $\mathbf{B}$, which leads the formation of conjugated diyne C along the reduction of the $\operatorname{Pd}(\mathrm{II})$ complex to $\operatorname{Pd}(0)$. The palladium species is later oxidized in the presence of $\mathrm{O}_{2}$ to give the initial Pd(II) species completing the cycle.

Due to our ongoing interest in tellurium structures, especially those involving $\pi$-interactions, the crystal and molecular structure of the bis-( $p$-tolylethynyl)telluride starting material was determined..$^{21}$ The tellurium atom is located on a crystallographic twofold axis with the C-Te-C angle being $92.23(15)^{\circ}$. The dihedral angle formed between the phenyl rings is $87.27(7)^{\circ}$ (Figure 3 ).

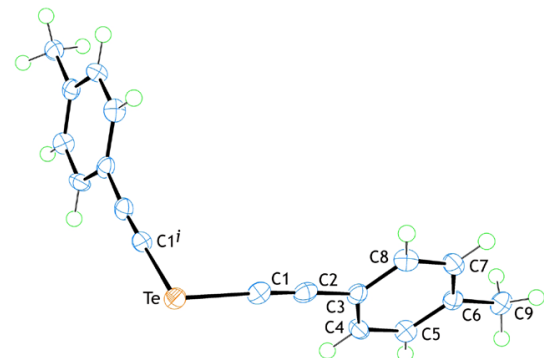

Figure 3. Molecular structure of bis( $p$-tolylethynyl)telluride showing numbering scheme and displacement ellipsoids at the $70 \%$ probability level. Symmetry operation i: -x, y, 3/2-z.

In the crystal structure, the telluride molecules are connected into supramolecular chains along the $b$ axis via $\mathrm{C}-\mathrm{H} . . . \pi$ interactions, as shown in Figure 4.

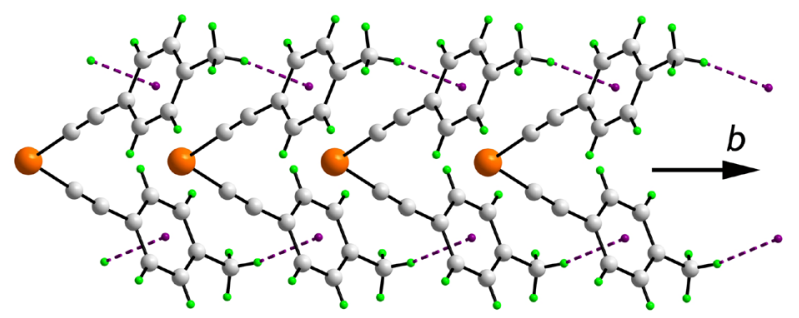

Figure 4. Supramolecular chain aligned along the $\mathrm{b}$ axis, sustained by C-H... $\pi$ interactions.

\section{Conclusions}

In summary, we demonstrated the synthesis of functionalized symmetrical 1,3-diyne systems through the palladium-catalyzed detelluration reaction of bis(arylethynyl) tellurides and bis(alkylethynyl)tellurides. The use of this methodology for the synthesis of more complex polyacetylenic compounds is currently under study in our laboratory.

\section{Experimental}

Proton nuclear magnetic resonance spectra $\left({ }^{1} \mathrm{H}\right.$ NMR) were obtained at $300 \mathrm{MHz}$. Spectra were recorded in $\mathrm{CDCl}_{3}$ solutions. Chemical shifts are reported in ppm, referenced to the solvent peak of $\mathrm{CDCl}_{3}$ or tetramethylsilane (TMS) as the external reference. Data are reported as follows: chemical shift $(\delta)$, multiplicity, coupling constant $(J)$ in Hertz and integrated intensity. Carbon-13 nuclear magnetic resonance spectra $\left({ }^{13} \mathrm{C}\right.$ NMR $)$ were obtained at $75 \mathrm{MHz}$. Spectra were 
recorded in $\mathrm{CDCl}_{3}$ solutions. Chemical shifts are reported in $\mathrm{ppm}$, referenced to the solvent peak of $\mathrm{CDCl}_{3}$. Abbreviations to denote the multiplicity of a particular signal are s (singlet), $d$ (doublet), $t$ (triplet), q (quartet), quint (quintet), sex (sextet) and $\mathrm{m}$ (multiplet). Column chromatography was performed using silica gel (230-400 mesh) following the methods described by Still et al. ${ }^{22}$ Thin layer chromatography (TLC) was performed using silica gel $\mathrm{F}_{254}, 0.25 \mathrm{~mm}$ thickness from Merck. For visualization, TLC plates were either placed under ultraviolet light, or stained with iodine vapor, or acidic vanillin. The following solvents were dried and purified by distillation from the reagents indicated: tetrahydrofuran from sodium with a benzophenone ketyl indicator. All other solvents were ACS or HPLC grade unless otherwise noted. Air and moisture-sensitive reactions were conducted in flame-dried or oven dried glassware equipped with tightly fitted rubber septa and under a positive atmosphere of dry nitrogen or argon. Reagents and solvents were handled using standard syringe techniques. Temperatures above room temperature were maintained by use of a mineral oil bath with an electrically heated coil connected to a controller.

General experimental procedure for preparing conjugated diyne compounds $2 \boldsymbol{a}-\boldsymbol{j}$

A suspension of bis(phenylethynyl)telluride (1a) (0.0824 g, $0.25 \mathrm{mmol}), \mathrm{PdOAc}_{2}(5.6 \mathrm{mg}, 10 \% \mathrm{~mol})$, triethylamine $(0.101 \mathrm{~g}, 1 \mathrm{mmol})$ and silver(I) acetate $(0.041 \mathrm{~g}, 0.25 \mathrm{mmol})$ in $5 \mathrm{~mL}$ of DMF was stirred at room temperature under air for $60 \mathrm{~min}$. The reaction mixture was then diluted with ethyl acetate $(25 \mathrm{~mL})$, and the organic layer was washed with a saturated solution of $\mathrm{NH}_{4} \mathrm{Cl}$ $(2 \times 10 \mathrm{~mL})$ and water $(2 \times 10 \mathrm{~mL})$, dried over $\mathrm{MgSO}_{4}$ and concentrated under vacuum. The crude product was purified by flash silica column chromatography using hexane as the eluent and subsequently characterized.

\section{Bis(phenylethynyl)telluride $(\mathbf{1 a})^{19}$}

Yield 86\%; ${ }^{1} \mathrm{H}$ NMR (300 $\left.\mathrm{MHz}, \mathrm{CDCl}_{3}\right) \delta(\mathrm{ppm})$ 7.30-7.38 (m, 6H), 7.47-7.56 (m, 4H). ${ }^{13} \mathrm{C} \mathrm{NMR}\left(\mathrm{CDCl}_{3}\right.$, 75.5 MHz,) $\delta$ (ppm) 73.7; 81.3; 121.6; 128.4; 129.5; 132.3; $\mathrm{EM} m / z(\%) 202$ (100), 200 (24), 150 (8), 101 (13), 88 (10).

\section{1,4-Bis(2,5-fluorophenylethynyl)telluride (1b)}

Red solid; mp 187-188 ${ }^{\circ} \mathrm{C}$. Yield $60 \%$; ${ }^{1} \mathrm{H} \mathrm{NMR}\left(\mathrm{CDCl}_{3}\right.$, $300 \mathrm{MHz}) \delta(\mathrm{ppm}) 6.84(\mathrm{dd}, 1 \mathrm{H}, J 5.2 \mathrm{~Hz}, J 13.0 \mathrm{~Hz}) 7.45$ $(\mathrm{dd}, 1 \mathrm{H}, J 8.0 \mathrm{~Hz}, J 15.3 \mathrm{~Hz}) .{ }^{13} \mathrm{C} \mathrm{NMR}\left(\mathrm{CDCl}_{3}, 75.5 \mathrm{MHz}\right)$ $\delta(\mathrm{ppm}) 165.4$ (d, $J 11.9 \mathrm{~Hz}, \mathrm{C}-\mathrm{F}), 161.8(\mathrm{~d}, J 46.4 \mathrm{~Hz}$, C-F), 135.1 (d, J 2.3 Hz, C-F), 11.76 (d, J $3.7 \mathrm{~Hz}, \mathrm{C}-\mathrm{F}$ ), 108.0 (d, J 4.2 Hz, C-F), 104.8 (d, J 19 Hz, C-F), 48.6 (2C), $29.4(2 \mathrm{C}) .{ }^{125} \mathrm{Te}$ NMR $\left(\mathrm{CDCl}_{3}, 95 \mathrm{MHz}\right) \delta$ (ppm) 374.1.
$\mathrm{EM} \mathrm{m/z( \% )} 274.2$ (100), 275.2 (16), 138.2 (15), 223.2 (6). IR (KBr) $v_{\max } / \mathrm{cm}^{-1}: 3440,3099,2139,1613,1586,1142$, 1096, 967, 855, 779, 729, 512, 498, 485. HRMS (ESI) calc. for $\mathrm{C}_{16} \mathrm{H}_{6} \mathrm{~F}_{4} \mathrm{TeNa}^{+}$426.9365; found 426.9368 .

\section{1,3-Bis(m-fluorophenylethynyl)telluride (1c)}

White solid; mp 129-130 ${ }^{\circ} \mathrm{C}$. Yield 58\%; ${ }^{1} \mathrm{HNMR}\left(\mathrm{CDCl}_{3}\right.$, $300 \mathrm{MHz}) \delta$ (ppm) 6.96-7.14 (m, 8H). ${ }^{13} \mathrm{C} \mathrm{NMR}\left(\mathrm{CDCl}_{3}\right.$, $75.5 \mathrm{MHz}$ ) $\delta$ (ppm) 44.5 (2C); 111.5 (2C); 116.4 (d, $J^{1} 21.1$ Hz, C-F); 118.8 (d, $\left.J^{2} 22.8 \mathrm{~Hz}, \mathrm{C}-\mathrm{F}\right) ; 124.4$ (d, $J^{3} 9.36 \mathrm{~Hz}$, C-F); 129.9 (d, $\left.J^{4} 3.03 \mathrm{~Hz}, \mathrm{C}-\mathrm{F}\right) ; 129.8$ (d, $\left.J^{5} 8.64 \mathrm{~Hz}, \mathrm{C}-\mathrm{F}\right)$; 162.2 (d, $\left.J^{6} 245.5 \mathrm{~Hz}, \mathrm{C}-\mathrm{F}\right) .{ }^{125} \mathrm{Te} \mathrm{NMR}\left(\mathrm{CDCl}_{3}, 95 \mathrm{MHz}\right)$ $\delta$ (ppm) 374.1. EM m/z (\%) 238.1 (100), 236.1 (13), 218.1 (6), 217.2 (5), 168.2 (4), 119.2 (16). IR (KBr) $v_{\max } / \mathrm{cm}^{-1}: 3440$, 3067, 2143, 1604, 1581, 1484, 1423, 1266, 1136, 941, 872, 784, 678, 571, 517, 457. HRMS (ESI) calc. for $\mathrm{C}_{16} \mathrm{H}_{8} \mathrm{~F}_{2} \mathrm{TeNa}^{+}$ 390.9553; found 390.9538 .

\section{1,4-Bis(p-tolylphenylethynyl)telluride $(\mathbf{1 d})^{23}$}

Yield 52\%; ${ }^{1} \mathrm{H}$ NMR (CDCl3, $\left.300 \mathrm{MHz}\right) \delta$ (ppm) 2.35 (s, 6H); 7.40 (d, J 7.8 Hz, 4H); $7.12(\mathrm{~d}, J 7.8 \mathrm{~Hz}, 4 \mathrm{H})$; ${ }^{13} \mathrm{C} \mathrm{NMR}\left(\mathrm{CDCl}_{3}, 75.5 \mathrm{MHz}\right.$,) $\delta$ (ppm) $21.6(2 \mathrm{C}) ; 73.5$ (2C); 81.6 (2C); 118.8 (2C); 129.2 (4C); 132.4 (4C); 139.5 (2C); EM $m / z$ (\%) 230 (100); 215 (17); 115 (17); 101 (15).

\section{1,4-Bis(p-fluorophenylethynyl)telluride (1e)}

White solid, mp 129-130 ${ }^{\circ} \mathrm{C}$. Yield 51\%; ${ }^{1} \mathrm{H}$ NMR $\left(\mathrm{CDCl}_{3}, 300 \mathrm{MHz}\right) \delta(\mathrm{ppm}) 6.91\left(\mathrm{t}, J^{1}\right.$ and $J^{2} 8.52 \mathrm{~Hz}$, $4 \mathrm{H}) ; 7.45\left(\mathrm{dd}, J^{1}\right.$ and $\left.J^{2} 5.37 \mathrm{~Hz}, 4 \mathrm{H}\right) .{ }^{13} \mathrm{C} \mathrm{NMR}\left(\mathrm{CDCl}_{3}\right.$, $75.5 \mathrm{MHz}) \delta(\mathrm{ppm}) 42.7$ (2C); $111.6(2 \mathrm{C}) ; 115.6(\mathrm{~d}$, $\left.J^{1} 22.0 \mathrm{~Hz}, \mathrm{C}-\mathrm{F}\right) ; 118.9\left(\mathrm{~d}, J^{2} 3.5 \mathrm{~Hz}, \mathrm{C}-\mathrm{F}\right) ; 134.2\left(\mathrm{~d}, \mathcal{J}^{3} 8.5 \mathrm{~Hz}\right.$, C-F); 162.9 (d, $\left.J^{4} 249.3 \mathrm{~Hz}, \mathrm{C}-\mathrm{F}\right) .{ }^{125} \mathrm{Te} \mathrm{NMR}\left(\mathrm{CDCl}_{3}, 95\right.$ MHz) $\delta$ (ppm) 364.5. EM m/z (\%) 264.2 (100), 265.1 (33), 249.2 (32), 233.6 (6), 231.4 (6), 221.1 (41), 220,2 (95), 201.5 (5.75), 200,4 (6) 132.3 (13), 117.1(5), 116.1 (8). IR (KBr) $v_{\text {max }} / \mathrm{cm}^{-1}: 3443,3099,3080,2143,1595,1503$, 1480, 1224, 1209, 1150, 1096, 838, 749, 533. HRMS (ESI) calc. for $\mathrm{C}_{16} \mathrm{H}_{8} \mathrm{~F}_{2} \mathrm{TeNa}^{+} 390.9553$, found 390.9538 .

\section{1,4-Bis(3-thyenyletynyl)telluride (1f)}

White solid, mp $100-101^{\circ} \mathrm{C}$. Yield $67 \%,{ }^{1} \mathrm{H}$ NMR $\left(\mathrm{CDCl}_{3}, 300 \mathrm{MHz}\right) \delta(\mathrm{ppm}) 7.14\left(\mathrm{dd}, J^{1}\right.$ and $J^{2} 1.14 \mathrm{~Hz}$, 2H); 7.25 (t, $J 3.0 \mathrm{~Hz}, 2 \mathrm{H}) ; 7.57\left(\mathrm{dd}, J^{1} 1.2 \mathrm{~Hz}\right.$ and $\left.J^{2} 1.14 \mathrm{~Hz}, 2 \mathrm{H}\right) .{ }^{13} \mathrm{C} \mathrm{NMR}\left(\mathrm{CDCl}_{3}, 75.5 \mathrm{MHz}\right) \delta(\mathrm{ppm})$ 42.7 (2C); 107.6 (2C); 122.1 (2C); 125.2 (2C); 130.1 (2C); 130.8 (2C). ${ }^{125} \mathrm{Te}$ NMR (CDCl, $\left.95 \mathrm{MHz}\right) \delta(\mathrm{ppm}) 364.4$. $\mathrm{EM} m / z$ (\%) 214.1 (100), 169,2 (19), 156.2 (4), 144.2 (6), 126.2 (4), 107.2 (11), $69.1(6), 45.0 . \mathrm{IR}(\mathrm{KBr}) \mathrm{v}_{\max } / \mathrm{cm}^{-1}$ : 3431, 3099, 2129, 1352, 1075, 940, 933, 865, 825, 783, $692,670,621,582$. HRMS (ESI) calc. for $\mathrm{C}_{12} \mathrm{H}_{6} \mathrm{~S}_{2} \mathrm{TeNa}^{+}$ 366.887 ; found 366.8855 . 


\section{1,4-Bis(4-methoxyphenylethynyl)telluride (1g) $)^{23}$}

Yield: $58 \%{ }^{1} \mathrm{H}$ NMR $\left(\mathrm{CDCl}_{3}, 300 \mathrm{MHz}\right) \delta(\mathrm{ppm}) 7.37$ $(\mathrm{d}, J 8.5 \mathrm{~Hz}, 4 \mathrm{H}) ; 6.67$ (dd, $J^{1} 2.6 \mathrm{~Hz}$ and $\left.J^{2} 8.5 \mathrm{~Hz}, 4 \mathrm{H}\right)$; $3.79(\mathrm{~s}, 6 \mathrm{H}) .{ }^{13} \mathrm{C} \mathrm{NMR}\left(\mathrm{CDCl}_{3}, 75.5 \mathrm{MHz},\right) \delta(\mathrm{ppm}) 20.9$ (2C), $55.2(2 \mathrm{C}), 111.2(2 \mathrm{C}), 115.0$ (4C), 129,8 (2C), 134.0 (4C), 143.1 (2C), 160.1 (2C), EM m/z (\%) 264.2 (100), 220.2 (95), 221.1 (41), 249.2 (32), 110.2 (21), 218.2 (10). IR (KBr) $v_{\max } / \mathrm{cm}^{-1}: 3437,3005,2984,2958,2936,2903$, 2891, 2838, 2824, 2154, 1463, 1440, 1432, 1427, 1348, 1273, 1187, 1082, 1069, 995, 976, 899, 880, 606, 590. HRMS (ESI) calc. for $\mathrm{C}_{18} \mathrm{H}_{14} \mathrm{O}_{2} \mathrm{TeNa}^{+}$, found 414.9851 .

\section{1,4-Bis(p-pentylphenylethynyl)telluride (1h)}

Yellow solid; mp 57-58 ${ }^{\circ} \mathrm{C}$. Yield: $50 \%$. ${ }^{1} \mathrm{H}$ NMR $\left(\mathrm{CDCl}_{3}, 300 \mathrm{MHz}\right) \delta(\mathrm{ppm}) 0.89(\mathrm{t}, J 6.6 \mathrm{~Hz}, 6 \mathrm{H}) ; 1.33$ $(\mathrm{m}, 8 \mathrm{H}) ; 1.60(\mathrm{~m}, 4 \mathrm{H}) ; 2.61(\mathrm{~m}, 4 \mathrm{H}) ; 7.14(\mathrm{~d}, J 8.0 \mathrm{~Hz}$, $4 \mathrm{H}), 7.39(\mathrm{~d}, J 7.8 \mathrm{~Hz}, 4 \mathrm{H}) .{ }^{13} \mathrm{C} \mathrm{NMR}\left(\mathrm{CDCl}_{3}, 75.5 \mathrm{MHz}\right)$ $\delta$ (ppm) 14.0 (2C); 22.5 (2C); 30.96 (2C); 35.9 (2C); 42.1(2C); 112.8 (2C); 120.1 (2C); 128.4 (2C); 132.1 (2C); $144.3(2 \mathrm{C}) .{ }^{125} \mathrm{Te} \mathrm{NMR}\left(\mathrm{CDCl}_{3}, 95 \mathrm{MHz}\right) \delta(\mathrm{ppm}) 357.6$. $\mathrm{EM} m / z$ (\%) 285.3 (100), 342.3 (92), 228.1 (61), 343.3 (25), 226.1 (12), 41.1 (9). IR (KBr) $v_{\max } / \mathrm{cm}^{-1}: 3435,3024$, 2955, 2929, 2855, 2141, 1503, 1466, 842, 825, 815, 723, 572, 530. HRMS (ESI) calc. for $\mathrm{C}_{26} \mathrm{H}_{30} \mathrm{TeNa}^{+} 495.1307$; found 495.1286 .

\section{1,4-Bis(methoxyethynyl)telluride (1i)}

Yellow solid, mp 44-45 ${ }^{\circ} \mathrm{C}$. Yield 78\%. ${ }^{1} \mathrm{H}$ NMR $\left(\mathrm{CDCl}_{3}, 300 \mathrm{MHz}\right) \delta(\mathrm{ppm}) 4.32(\mathrm{~s}, 4 \mathrm{H}) ; 3.39$ (s, 6H). ${ }^{13} \mathrm{C} \mathrm{NMR}\left(\mathrm{CDCl}_{3}, 75.5 \mathrm{MHz},\right) \delta(\mathrm{ppm}) 40.2(2 \mathrm{C}) ; 57.4$ (2C); 60.6 (2C); 109.9 (2C). EM m/z (\%) 69.0 (100), 77.1 (14), 78.1(14), 63.1 (13), 51.1 (11), 53.0 (8).

\section{1,4-Bis(butylethynyl)telluride (1j) $)^{24}$}

Yellow oil; ${ }^{1} \mathrm{H}$ NMR $\left(\mathrm{CDCl}_{3}, 300 \mathrm{MHz}\right) \delta(\mathrm{ppm}) 0.90$ (t, J 7.2 Hz, 6H); 1.36-1.57 (m, 8H); $2.50(\mathrm{t}, J 6.9 \mathrm{~Hz}, 4 \mathrm{H})$. ${ }^{13} \mathrm{C} \mathrm{NMR}\left(\mathrm{CDCl}_{3}, 75.5 \mathrm{MHz}\right) \delta(\mathrm{ppm}) 13.6(2 \mathrm{C}) ; 20.7$ (2C); 21.9 (2C); 30.8 (4C); 114.3 (2C).

\section{General procedure to homocoupling reaction}

A suspension of bis(phenylethynyl)telluride (1a) (0.0824 g; $0.25 \mathrm{mmol}), \mathrm{Pd}(\mathrm{OAc})_{2}(0.0056 \mathrm{~g}, 10 \% \mathrm{~mol})$, triethylamine $(0.101 \mathrm{~g} ; 1 \mathrm{mmol})$ and silver acetate $(0.041 \mathrm{~g}$, $0.5 \mathrm{mmol}$ ) in $5 \mathrm{~mL}$ of DMF was stirred at room temperature, under air atmospheric by $60 \mathrm{~min}$. The reaction mixture was diluted with ethyl acetate $(25 \mathrm{~mL})$, and the organic layer washed with $\mathrm{NH}_{4} \mathrm{Cl}(2 \times 10 \mathrm{~mL})$, dried over $\mathrm{MgSO}_{4}$ and concentrated under reduced pressure. The crude product was purified by flash silica column chromatography using hexane as eluent and subsequently characterized.

\section{1,4-Bis(phenylbuta-1,3-diyne $(2 a)^{25}$}

Yield $86 \% .{ }^{1} \mathrm{H}$ NMR $\left(\mathrm{CDCl}_{3}, 300 \mathrm{MHz}\right) \delta(\mathrm{ppm})$ 7.30-7.38 (m, 6H), 7.47-7.56 (m, 4H). ${ }^{13} \mathrm{C} \mathrm{NMR}\left(\mathrm{CDCl}_{3}\right.$, $75.5 \mathrm{MHz},) \delta(\mathrm{ppm}) 73.7 ; 81.3 ; 121.6 ; 128.4 ; 129.5 ; 132.3$.

\section{1,4-Bis(2,4-difluorophenyl)buta-1,3-diyne (2b)}

White solid; mp 187-188 ${ }^{\circ} \mathrm{C}$. Yield $60 \%$. ${ }^{1} \mathrm{H}$ NMR $\left(\mathrm{CDCl}_{3}, 300 \mathrm{MHz}\right) \delta(\mathrm{ppm})$ 6.83-6.90 (m, 4H); 7.54-7.46 (m, 2H). ${ }^{13} \mathrm{C} \mathrm{NMR}\left(\mathrm{CDCl}_{3}, 75.5 \mathrm{MHz}\right) \delta(\mathrm{ppm}) 29.7$ (2C); 74.8 (2C); 104.6 (d, J 1.3 Hz, C-F); 111.9 (d, J 25.7, C-F); 135.3 (d, J 9.9 Hz, C-F); 160.1 (d, J 196.0 Hz, C-F); 165.6 (d, J 53.8 Hz, C-F). IR (KBr) $v_{\max } / \mathrm{cm}^{-1}: 3439,3101,2955$, 2924, 2854, 1730, 1609, 1582, 1496, 1468, 1435, 1299, $1268,1143,1098,965,857,852,812,741,710,612,601$, 508, 475, 429. Microanalysis Calc. $70.25 \%$ C, $2.46 \%$ H; found $70.44 \% \mathrm{C} ; 2.54 \% \mathrm{H}$.

\section{1,3-Bis(m-fluorophenyl)buta-1,3-diyne (2c $)^{26}$}

Yield 65\%; ${ }^{1} \mathrm{H} \mathrm{NMR}\left(\mathrm{CDCl}_{3}, 300 \mathrm{MHz}\right) \delta(\mathrm{ppm}) 7.22-$ $7.29(\mathrm{~m}, 8 \mathrm{H}) .{ }^{13} \mathrm{C} \mathrm{NMR}\left(\mathrm{CDCl}_{3}, 75.5 \mathrm{MHz},\right) \delta(\mathrm{ppm}) 74.4$ (2C); 80.6 (2C); 116.9 (d, $\left.J^{1} 21.0 \mathrm{~Hz}, \mathrm{C}-\mathrm{F}\right) ; 119.2$ (d, $J^{2}$ $22.9 \mathrm{~Hz}, \mathrm{C}-\mathrm{F}) ; 123.4$ (d, $\left.J^{3} 9.5 \mathrm{~Hz}, \mathrm{C}-\mathrm{F}\right) ; 128.4$ (d, J $J^{4} 3.1 \mathrm{~Hz}$, C-F); 130.1 (d, J $\left.{ }^{5} 8.6 \mathrm{~Hz}, \mathrm{C}-\mathrm{F}\right) ; 162.2$ (d, J $\left.{ }^{6} 245.8 \mathrm{~Hz}, \mathrm{C}-\mathrm{F}\right)$.

\section{1,4-Bis(p-tolyl)buta-1,3-diyne (2d $)^{27}$}

Yield 52\%. ${ }^{1} \mathrm{H}$ NMR $\left(\mathrm{CDCl}_{3}, 300 \mathrm{MHz},\right) \delta(\mathrm{ppm})$ 2.35 (s, 6H); $7.40(\mathrm{~d}, J 7.8 \mathrm{~Hz}, 4 \mathrm{H}) ; 7.12(\mathrm{~d}, J 7.8 \mathrm{~Hz}$, $4 \mathrm{H}) ;{ }^{13} \mathrm{C} \mathrm{NMR}\left(\mathrm{CDCl}_{3}, 75.5 \mathrm{MHz},\right) \delta$ (ppm) $21.6(2 \mathrm{C})$; 73.5 (2C); 81.6 (2C); 118.8 (2C); 129.2 (4C); 132.4 (4C); $139.5(2 \mathrm{C})$.

\section{1,4-Bis(p-fluorophenyl)buta-1,3-diyne (2e $)^{28}$}

Yield 47\%. ${ }^{1} \mathrm{H}$ NMR $\left(\mathrm{CDCl}_{3}, 300 \mathrm{MHz}\right) \delta(\mathrm{ppm}) 7.03$ (dd, $1 \mathrm{H}, J 7.8 \mathrm{~Hz}, J 9.7 \mathrm{~Hz}$ ); $7.51(\mathrm{ddd}, J 2.2 \mathrm{~Hz}, J 5.3 \mathrm{~Hz}$, $J 6.9 \mathrm{~Hz}) .{ }^{13} \mathrm{C} \mathrm{NMR}\left(\mathrm{CDCl}_{3}, 75.5 \mathrm{MHz}\right) \delta(\mathrm{ppm}) 73.5(2 \mathrm{C})$; 80.4 (2C); 115.9 (d, $\left.J^{1} 22.1 \mathrm{~Hz}, \mathrm{C}-\mathrm{F}\right) ; 117.8\left(\mathrm{~d}, J^{2} 3.56 \mathrm{~Hz}\right.$, C-F); 134.5 (d, $\left.J^{3} 8.50 \mathrm{~Hz}, \mathrm{C}-\mathrm{F}\right) ; 163.0$ (d, J $\left.J^{4} 250.1 \mathrm{~Hz}, \mathrm{C}-\mathrm{F}\right)$.

\section{1,3-Bis(3-thyenyl)buta-1,3-diyne (2f) ${ }^{28}$}

Yield 44\%; ${ }^{1} \mathrm{H}$ NMR $\left(\mathrm{CDCl}_{3}, 300 \mathrm{MHz},\right) \delta(\mathrm{ppm}) 7.12$ (d, J 5.0 Hz, 2H); 7.25-7.28 (m, 2H); $7.58(\mathrm{~d}, J 2.9 \mathrm{~Hz}, 2 \mathrm{H})$. ${ }^{13} \mathrm{C} \mathrm{NMR}\left(\mathrm{CDCl}_{3}, 75.5 \mathrm{MHz}\right) \delta(\mathrm{ppm}) 73.6(2 \mathrm{C}) ; 121.0$ (2C); 125.6 (4C); 131.2 (4C).

\section{1,4-Bis(p-methoxyphenyl)buta-1,3-diyne (2g $)^{28}$}

Yield 39\%; ${ }^{1} \mathrm{H}$ NMR $\left(\mathrm{CDCl}_{3}, 300 \mathrm{MHz}\right) \delta(\mathrm{ppm}) 7.42$ (d, $J 8.5 \mathrm{~Hz}, 4 \mathrm{H}$ ); 6.69 (dd, $J^{1} 2.5 \mathrm{~Hz}$ and $J^{2} 8.5 \mathrm{~Hz}, 4 \mathrm{H}$ ); $3.80(\mathrm{~s}, 6 \mathrm{H}) .{ }^{13} \mathrm{C}$ NMR $\left(\mathrm{CDCl}_{3}, 75.5 \mathrm{MHz}\right) \delta(\mathrm{ppm}) 55.2$ (2C); 55.2 (2C); 67.9 (2C); 80.8 (2C); 111.4 (2C); 115.2 (4C); 134.3 (4C); 160,0 (2C). 
1,4-Bis(p-penthylphenyl)buta-1,3-diyne (2h $)^{29}$

Yield 38\%; ${ }^{1} \mathrm{H} \mathrm{NMR}\left(\mathrm{CDCl}_{3}, 300 \mathrm{MHz}\right) \delta$ (ppm) 0.87 $(\mathrm{m}, 6 \mathrm{H}) ; 1.30(\mathrm{~m}, 8 \mathrm{H}) ; 1.59(\mathrm{t}, J 7.0 \mathrm{~Hz}, 4 \mathrm{H}) ; 2.59(\mathrm{~m}, 4 \mathrm{H})$; $7.12\left(\mathrm{dd}, J^{1} 3.8 \mathrm{~Hz}\right.$ and $\left.J^{2} 7.9 \mathrm{~Hz}, 4 \mathrm{H}\right) ; 7.40(\mathrm{t}, J 8.6 \mathrm{~Hz}$, $4 \mathrm{H}) .{ }^{13} \mathrm{C} \mathrm{NMR}\left(\mathrm{CDCl}_{3}, 75.5 \mathrm{MHz}\right) \delta(\mathrm{ppm}) 13.9(2 \mathrm{C}) ; 22.5$ (2C); 31.4 (4C); 35.9 (2C); 73.5 (2C); 83.9 (2C); 119.0 (2C); 128.5 (4C); 132.4 (4C); 144.4 (2C).

\section{1,6-Dimethoxy-2,4-hexadiyne (2i $)^{29}$}

Yield 21\%; ${ }^{1} \mathrm{H} \mathrm{NMR}\left(\mathrm{CDCl}_{3}, 300 \mathrm{MHz}\right) \delta$ (ppm) 3.39 $(\mathrm{s}, 6 \mathrm{H}) ; 4.18(\mathrm{~s}, 4 \mathrm{H}) .{ }^{13} \mathrm{C} \mathrm{NMR}\left(\mathrm{CDCl}_{3}, 75.5 \mathrm{MHz}\right) \delta(\mathrm{ppm})$ 57.4 (2C); 59.8 (2C); 70.1 (2C); 75.1 (2C).

\section{Supplementary Information}

Supplementary data are available free of charge at http://jbcs.sbq.org.br as PDF file.

\section{Acknowledgments}

The authors thank FAPESP (2007/59404-2) and CNPq for fellowships to H. A. S. and J. M. P. (300.613/2007-5 and $142.741 / 2008$, respectively) for financial support.

\section{References}

1. Patai, S.; Rappoport, Z.; The Chemistry of Organic Selenium and Tellurium Compounds, Wiley: Chichester, 1986, vol. 1; Irgolic, K. Y. In Houben-Weyl Methoden der Organischen Chemie; Müller, E., ed., $4^{\text {th }}$ ed., vol. E12b, G. Thieme: Stuttgart, 1990; Petragnani, N.; Stefani, H. A. Tetrahedron 2005, 61, 1613; Petragnani, N.; Stefani, H. A. In Tellurium in Organic Chemistry; Second, Updated and Enlarged Edition, Academic Press, Elsevier: Amsterdam, 2007.

2. Bergman, J.; Carlsson, R.; Sjöberg, B.; Org. Synth. 1977, 57, 18; Bergman, J.; Tetrahedron 1972, 28, 3323; Bergman, J.; Engman, L.; Tetrahedron 1980, 36, 1275.

3. Barton, D. H. R.; Ozbalik, N.; Ramesh, M.; Tetrahedron Lett. 1988, 29, 3533.

4. Stang, P. J.; Diederich, F.; Modern Acetylene Chemistry, VCH: Weinheim, Germany, 1995, p. 506; Patai, S.; The Chemistry of the Carbon-Carbon Triple Bond, Wiley-Interscience: London, 1978.

5. Damle, S. V.; Seomoon, D.; Lee, P. H.; J. Org. Chem. 2003, 68, 7085.

6. For general reviews on coupling reactions between $s p$-carbon centers, see: Sonogashira, K. In Comprehensive Organic Synthesis; Trost, B. M.; Fleming, I., eds.; Pergamon: Oxford,
1991, Vol. 3, pp. 551-561; Siemsen, P.; Livingston, R. C.; Diederich, F.; Angew. Chem., Int. Ed. 2000, 39, 2632.

7. Bohlmann, F.; Burkhardt, T.; Zdero, C.; Naturally Occurring Acetylenes, Academic Press: London, 1973, p. 548.

8. Mayer, S. F.; Steinreiber, A.; Orru, R. V. A.; Faber, K.; J. Org. Chem. 2002, 67, 9115.

9. Stefani, H. A.; Costa, I. M.; Zeni, G.; Tetrahedron Lett. 1999, 40, 9215.

10. Zeni, G.; Panatieri, R. B.; Lissner, E.; Menezes, P. H.; Braga, A. L.; Stefani, H. A.; Org. Lett. 2001, 3, 819.

11. Stüts, A.; Angew. Chem., Int. Ed. 1987, 26, 320.

12. Diederich, F.; Rubin, Y.; Angew. Chem., Int. Ed. 1992, 31, 1101; Tour, J. M.; Chem. Rev. 1996, 96, 537; Martin, R. E.; Diederich, F.; Angew. Chem., Int. Ed. 1999, 38, 1350.

13. Kimball, D. B.; Haley, M. M.; Mitchell, R. H.; Ward, T. J.; Bandyopadhyay, S.; Williams, R. V; Armantrout, J. A.; J. Org. Chem. 2002, 67, 8798.

14. O'Connor, M. J.; Haley, M. M.; Org. Lett. 2004, 6, 2385.

15. Stefani, H. A.; Guarezemini, A. S.; Cella, R.; Tetrahedron 2010, 66, 7871 .

16. Eglinton, G.; Galbraith, A. R.; J. Chem. Soc. 1959, 889.

17. Hay, A. S.; J. Org. Chem. 1962, 27, 3320.

18. Taylor, R. J. K.; Organocopper Reagents, Oxford University Press: New York, 1994, p. 352.

19. Engman, L.; Stern, D.; Organometallics 1993, 12, 1445.

20. Punniyamurthy, T.; Velusamy, S.; Iqbal, J.; Chem. Rev. 2005, $105,2329$.

21. Caracelli, I.; Zukerman-Schpector, J.; Pena, J. M.; Stefani, H. A.; Tiekink, E. R. T.; Acta Crystallogr., Sect. E: Struct. Rep. Online 2010, 66, o685.

22. Still, W.C.; Kahn, M.; Mitra, A.; J. Org. Chem. 1978, 43, 2923.

23. Murai, T.; Imaeda, K.; Kajita, S.; Kimura, K.; Ishihara, K.; Kato, S.; Phosphorus, Sulfur Silicon Relat. Elem. 1992, 67, 239.

24. Citeau,G. A.; Giolando, D. M.; J. Organomet. Chem. 2001, 625, 23.

25. Coste, A.; Couty, F.; Evano, G.; Synthesis 2010, 9, 1500.

26. Jiang, H-F.; Liu, H-L.; Zhan, H-Y.; Zhou, L.; Chin. J. Chem. 2007, 25, 1413.

27. Chen, S-N.; Wu, W-Y.; Tsai, F-Y.; Green Chem. 2009, 11, 269.

28. Wang, D.; Li, J.; Li, N.; Gao, T.; Hou, S.; Chen, B.; Green Chem. 2010, 12, 45.

29. Stefani, H. A.; Singh, F. V.; Amaral, M. F. Z. J.; Tetrahedron Lett. 2009, 50, 2636.

Submitted: November 11, 2010 Published online: April 5, 2011

FAPESP has sponsored the publication of this article. 


\section{Supplementary Information}

\section{Synthesis of 1,3-Diynes via Detelluration of Bis(ethynyl)tellurides}

\section{Hélio A. Stefani, ${ }^{*, a}$ Jesus M. Pena, ${ }^{a}$ Julio Zuckerman-Schpector ${ }^{b}$ and Edward R. T. Tiekink ${ }^{c}$}

${ }^{a}$ Faculdade de Ciências Farmacêuticas, Universidade de São Paulo, São Paulo-SP, Brazil

${ }^{b}$ Departamento de Química, Universidade Federal de São Carlos, 13565-905 São Carlos-SP, Brazil

'Department of Chemistry, University of Malaya, Kuala Lumpur 50603, Malaysia

Table S1. Chemical shifts for ${ }^{127} \mathrm{Te}$

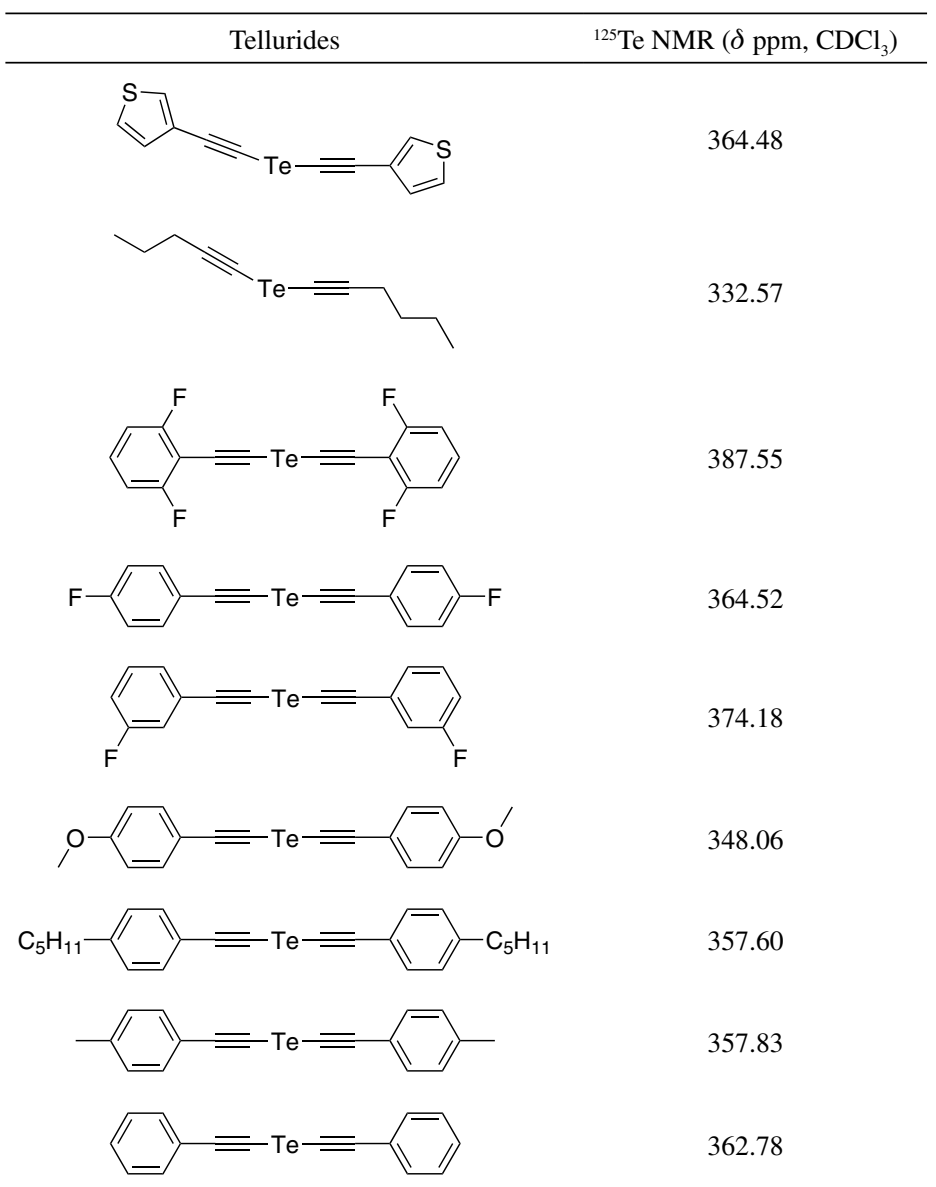

All spectra were obtained using diphenyl ditelluride as internal standard. $\mathrm{Te}_{2} \mathrm{Ph}_{2}$ $\left(\mathrm{CDCl}_{3}, \delta\left({ }^{125} \mathrm{Te}\right)=421.8 \mathrm{ppm}\right)$. 

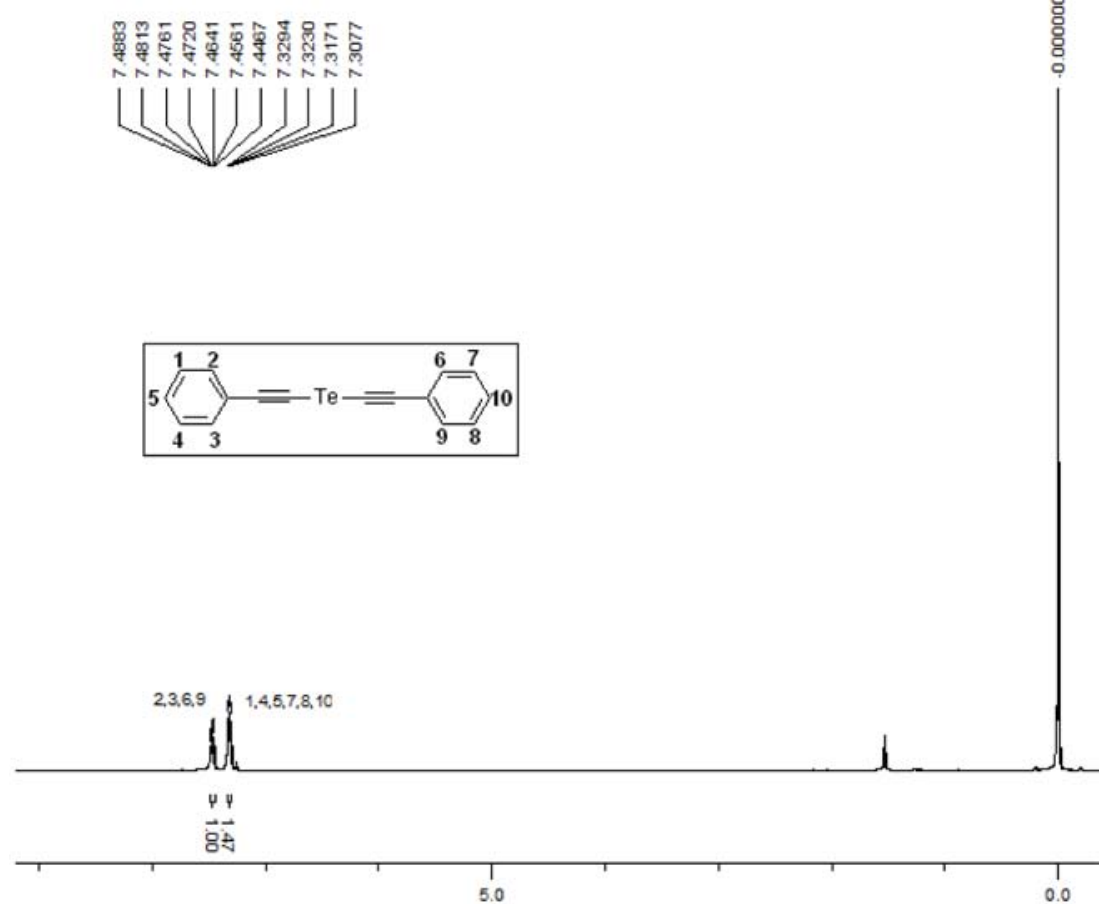

Figure S1. ${ }^{1} \mathrm{H}$ NMR $\left(\mathrm{CDCl}_{3}, 300 \mathrm{MHz}\right)$ of $\mathbf{1 a}$.
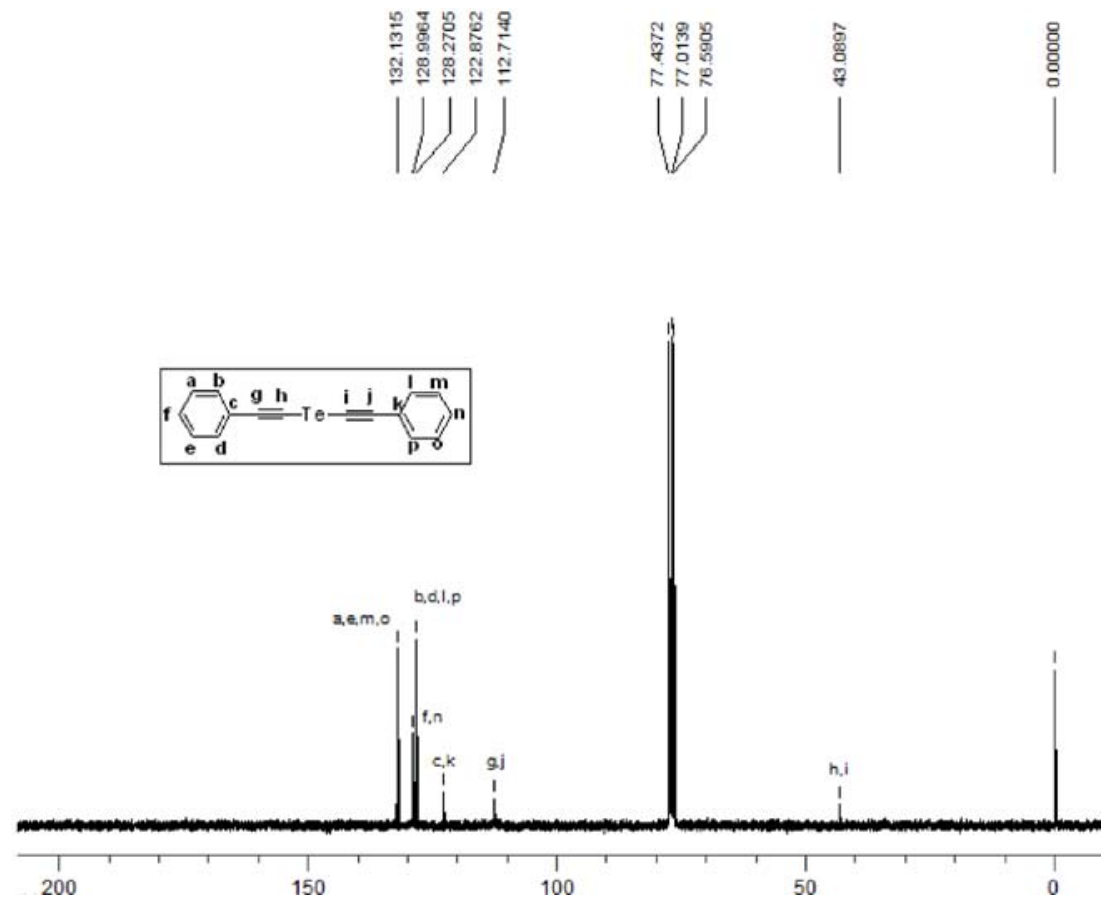

Figure S2. ${ }^{13} \mathrm{C}$ NMR $\left(\mathrm{CDCl}_{3}, 300 \mathrm{MHz}\right)$ of $\mathbf{1 a}$. 


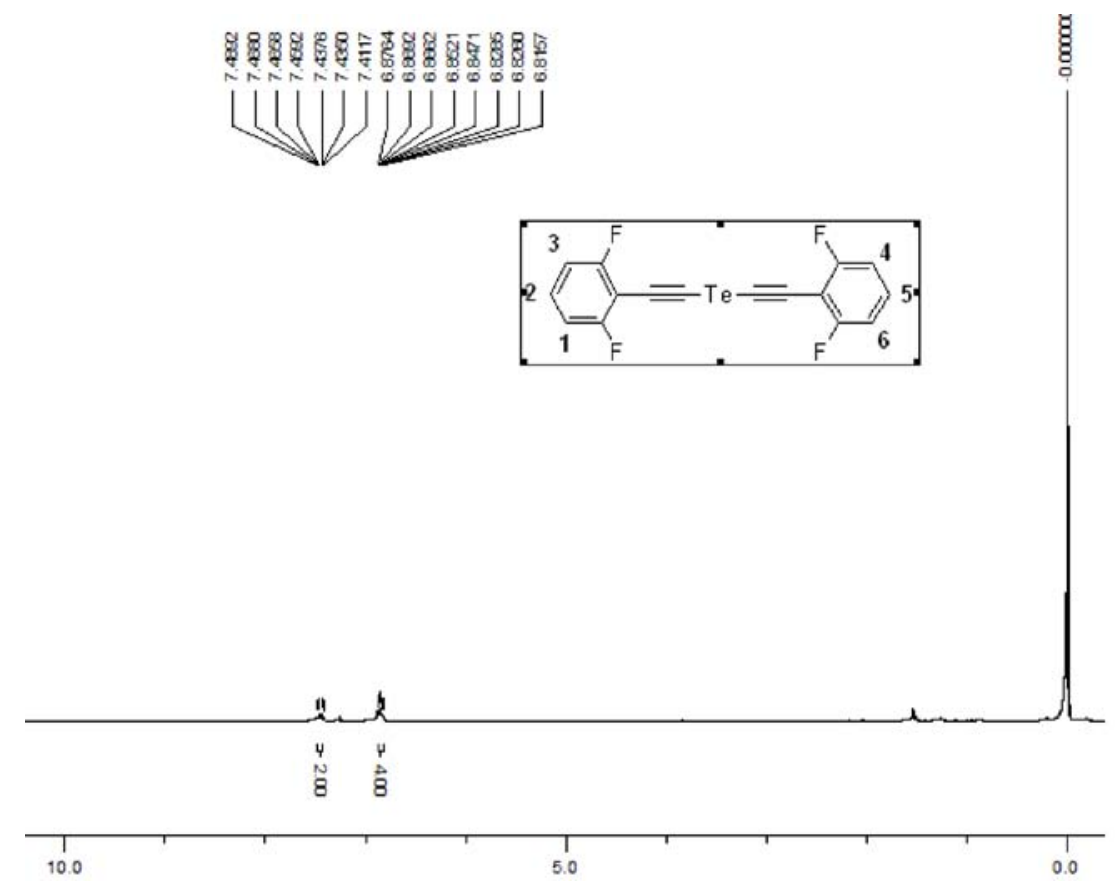

Figure S3. ${ }^{1} \mathrm{H}$ NMR $\left(\mathrm{CDCl}_{3}, 300 \mathrm{MHz}\right)$ of $\mathbf{1} \mathbf{b}$.
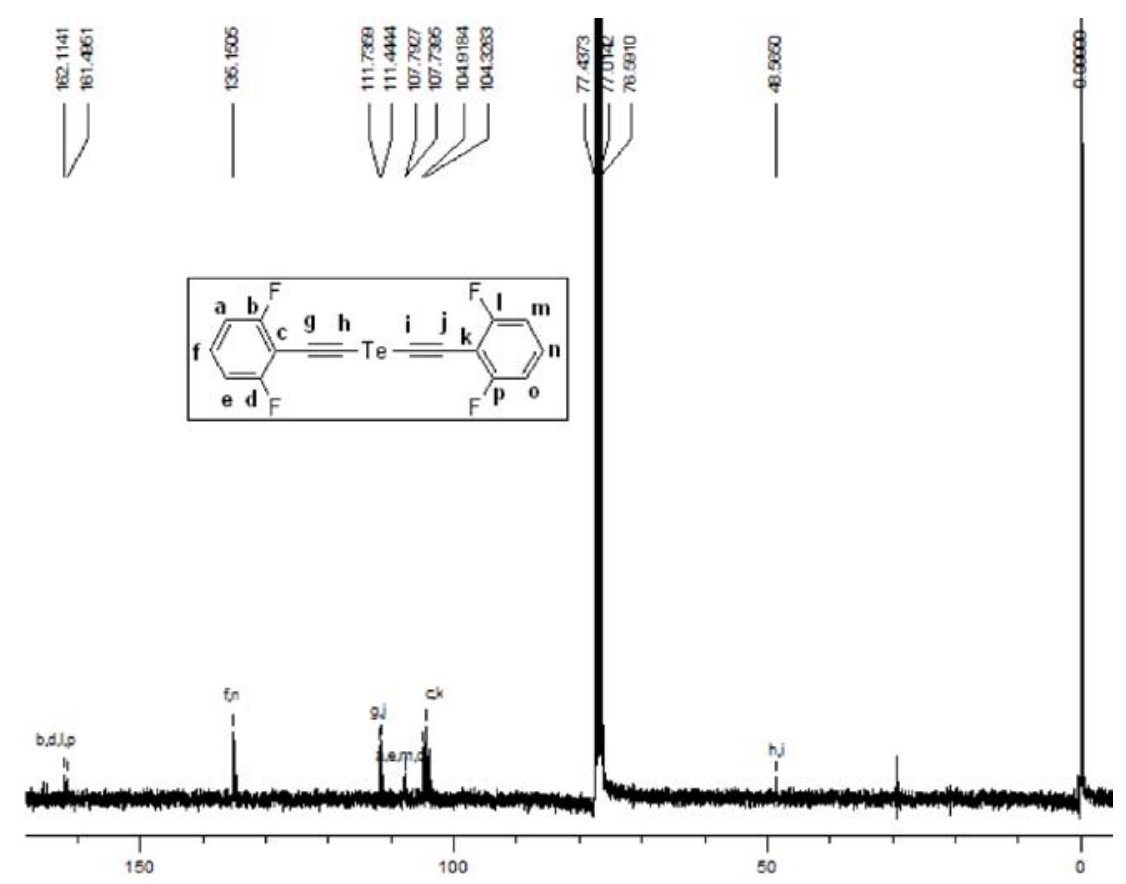

Figure S4. ${ }^{13} \mathrm{C}$ NMR $\left(\mathrm{CDCl}_{3}, 300 \mathrm{MHz}\right)$ of $\mathbf{1 b}$. 

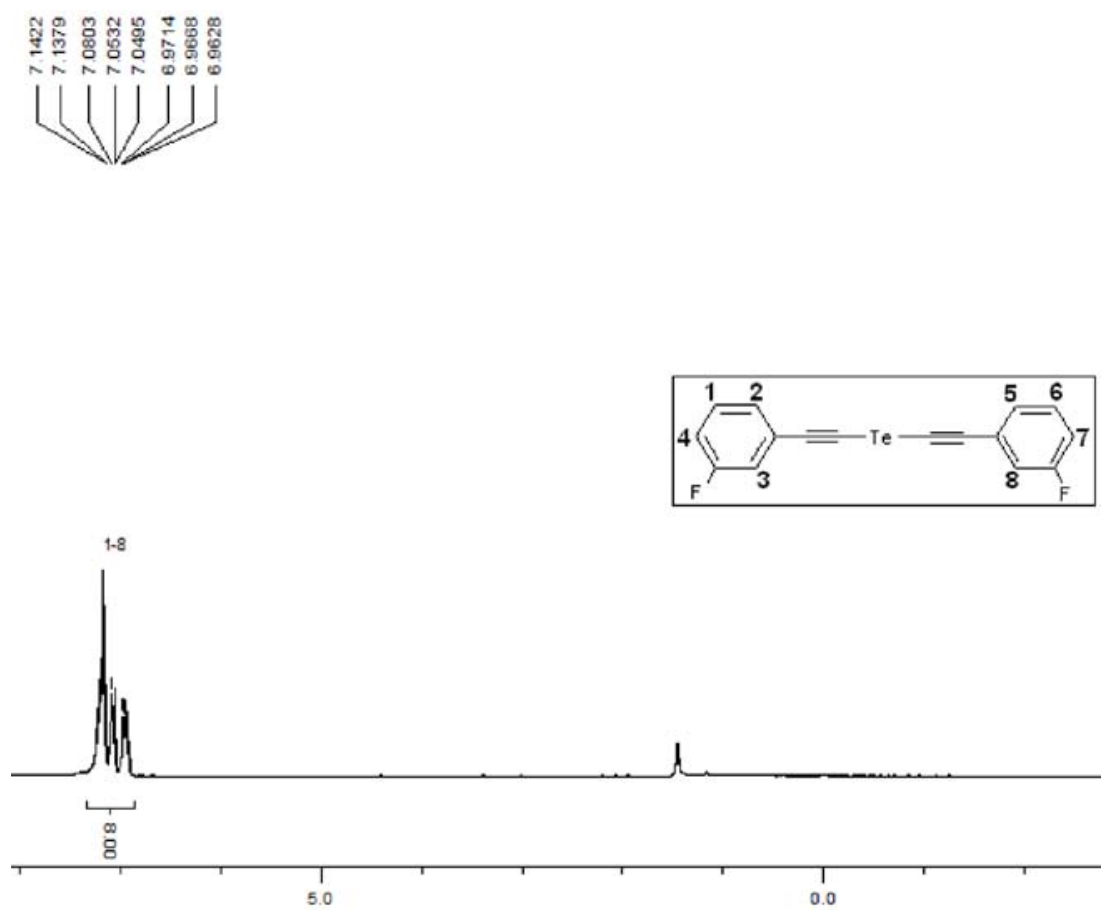

Figure S5. ${ }^{1} \mathrm{H}$ NMR $\left(\mathrm{CDCl}_{3}, 300 \mathrm{MHz}\right)$ of $\mathbf{1 c}$.
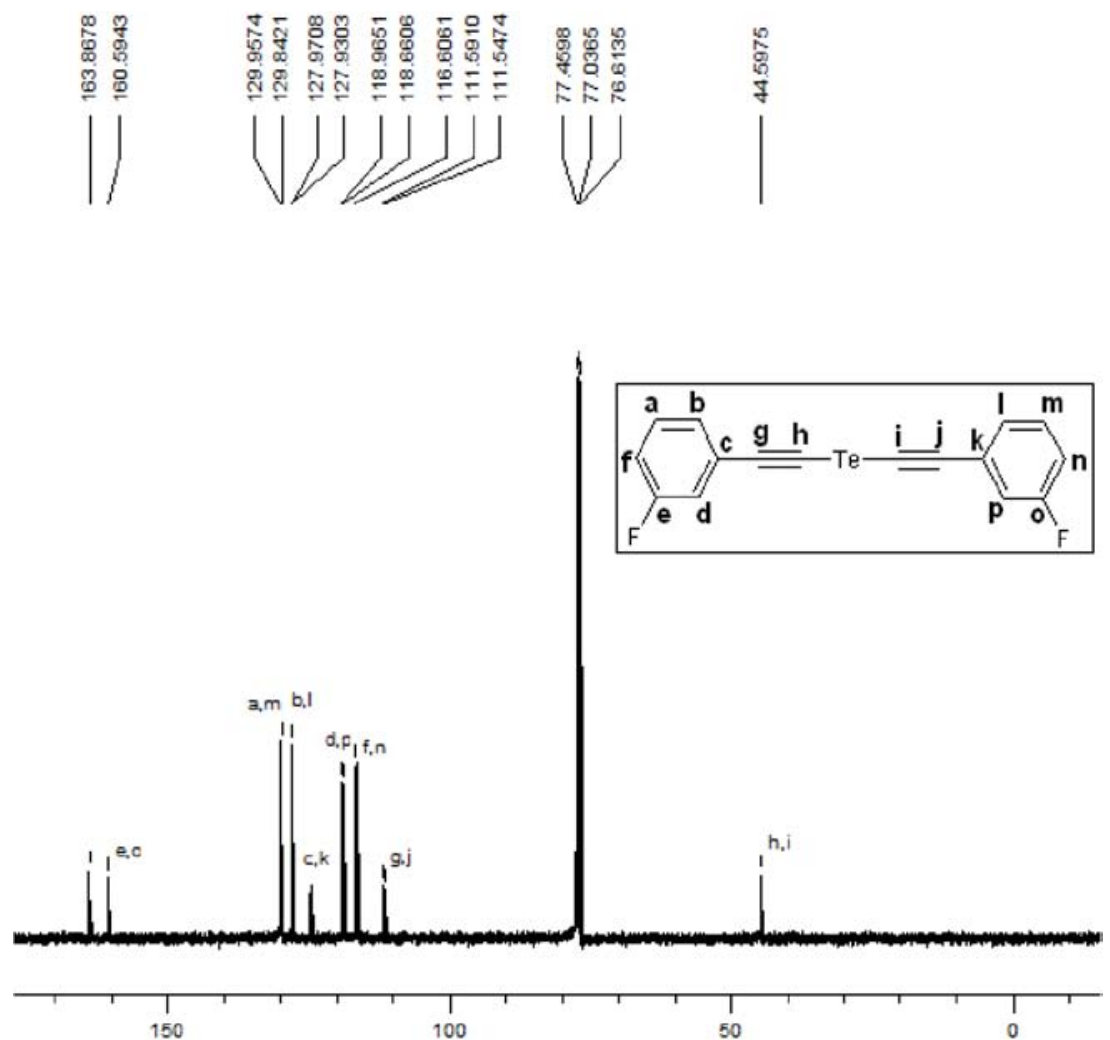

Figure S6. ${ }^{13} \mathrm{C}$ NMR $\left(\mathrm{CDCl}_{3}, 300 \mathrm{MHz}\right)$ of $\mathbf{1 c}$. 

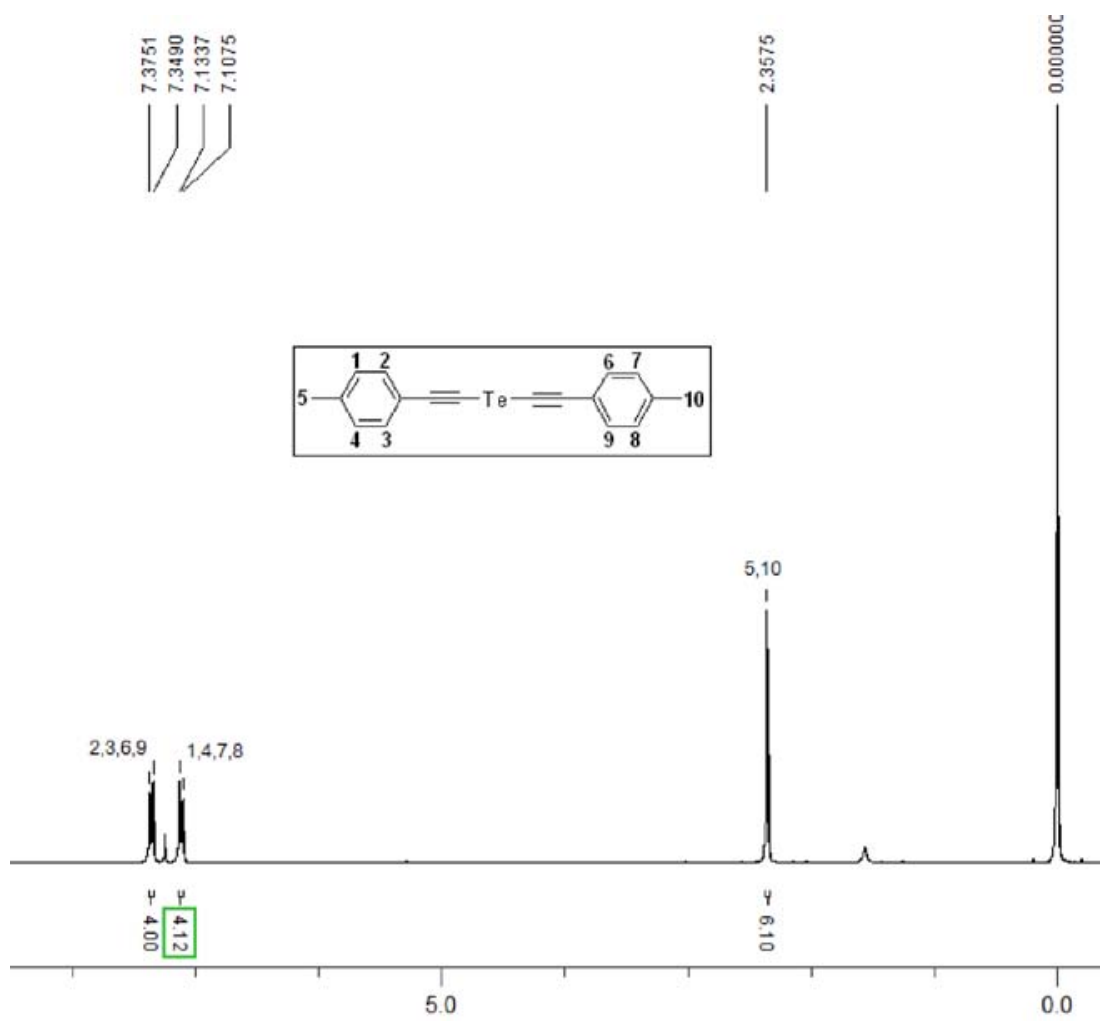

Figure S7. ${ }^{1} \mathrm{H} \mathrm{NMR}\left(\mathrm{CDCl}_{3}, 300 \mathrm{MHz}\right)$ of $\mathbf{1 d}$.
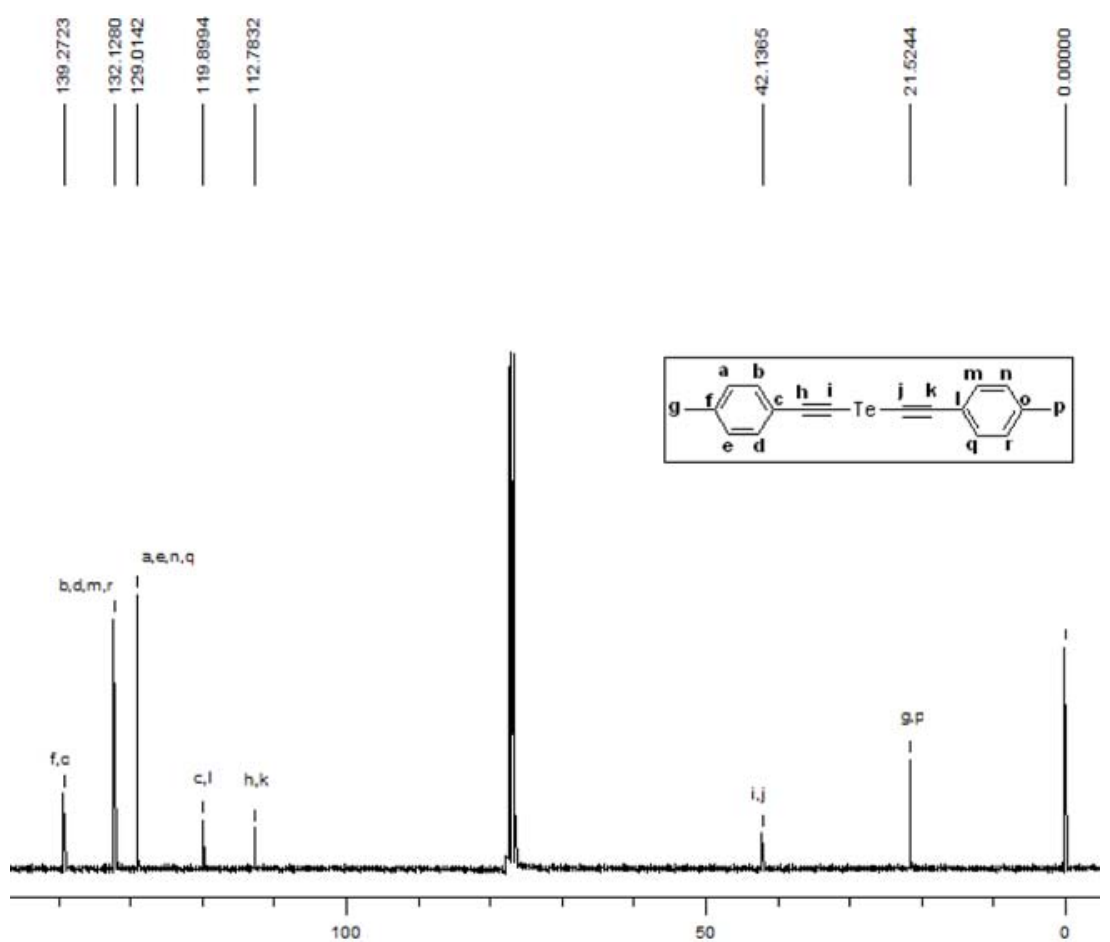

Figure S8. ${ }^{13} \mathrm{C} \mathrm{NMR}\left(\mathrm{CDCl}_{3}, 300 \mathrm{MHz}\right)$ of $\mathbf{1 d}$. 

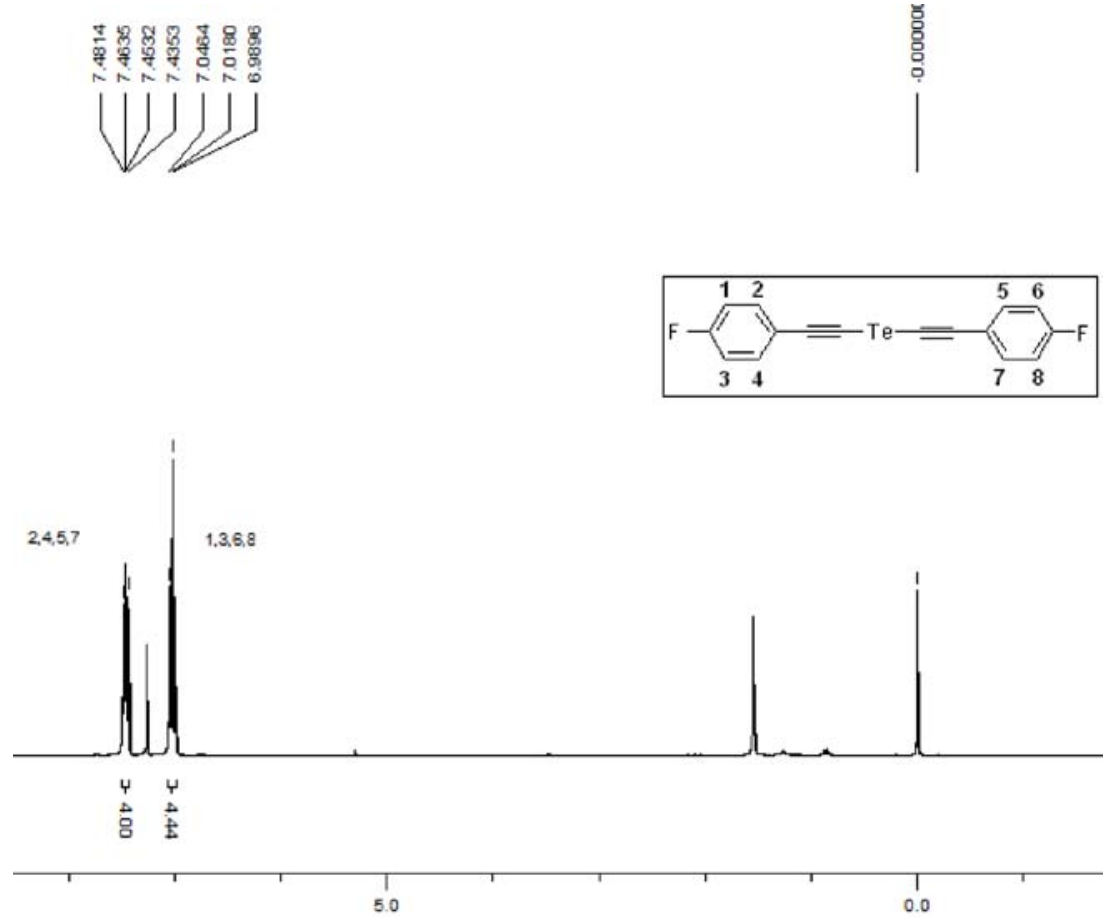

Figure S9. ${ }^{1} \mathrm{H} \mathrm{NMR}\left(\mathrm{CDCl}_{3}, 300 \mathrm{MHz}\right)$ of $\mathbf{1 e}$
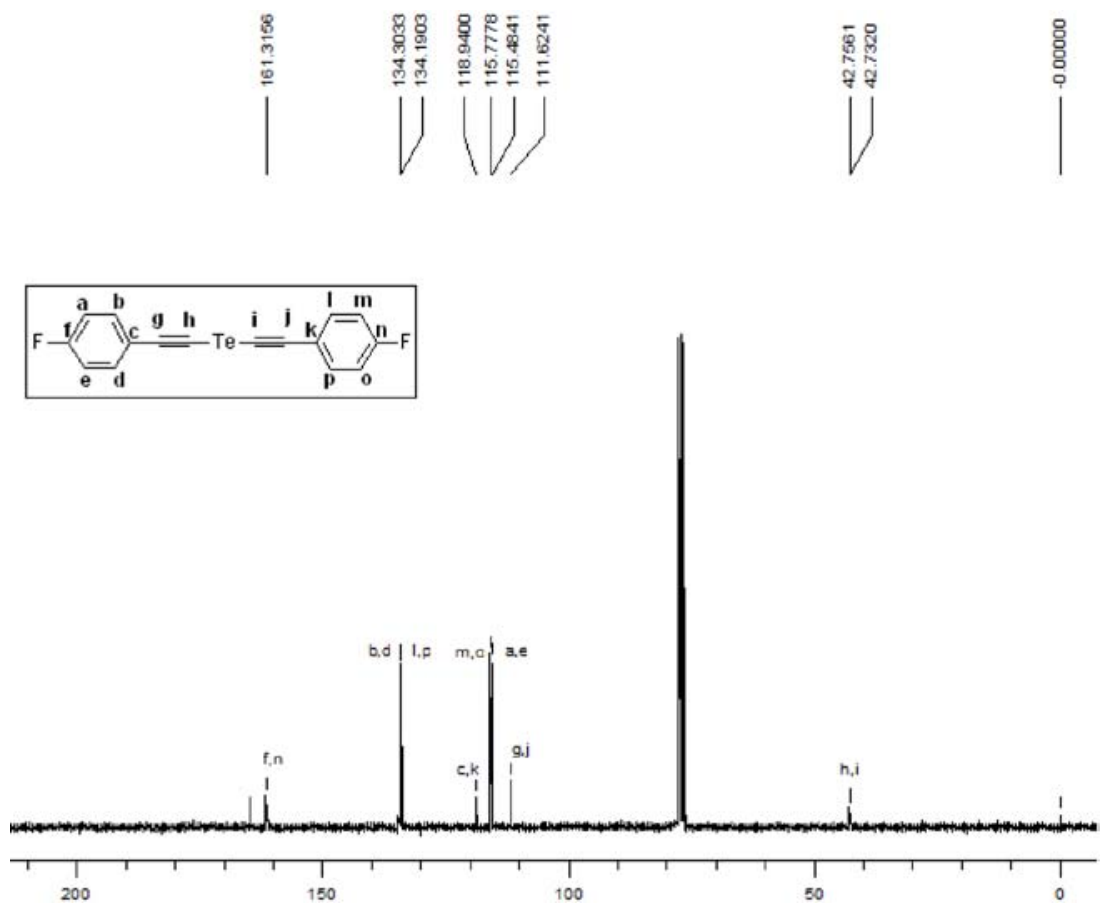

Figure S10. ${ }^{13} \mathrm{C}$ NMR $\left(\mathrm{CDCl}_{3}, 300 \mathrm{MHz}\right)$ of $1 \mathrm{e}$. 

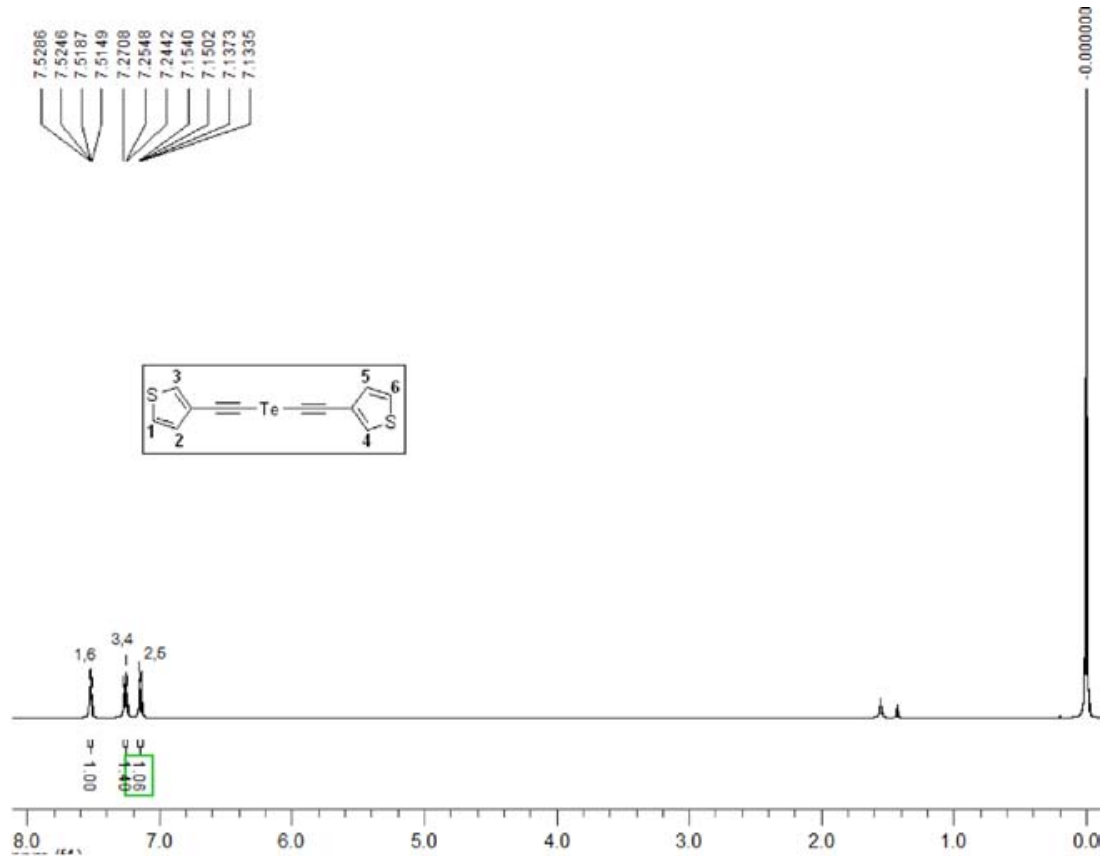

Figure S11. ${ }^{1} \mathrm{H} \mathrm{NMR}\left(\mathrm{CDCl}_{3}, 300 \mathrm{MHz}\right)$ of $\mathbf{1 f}$.
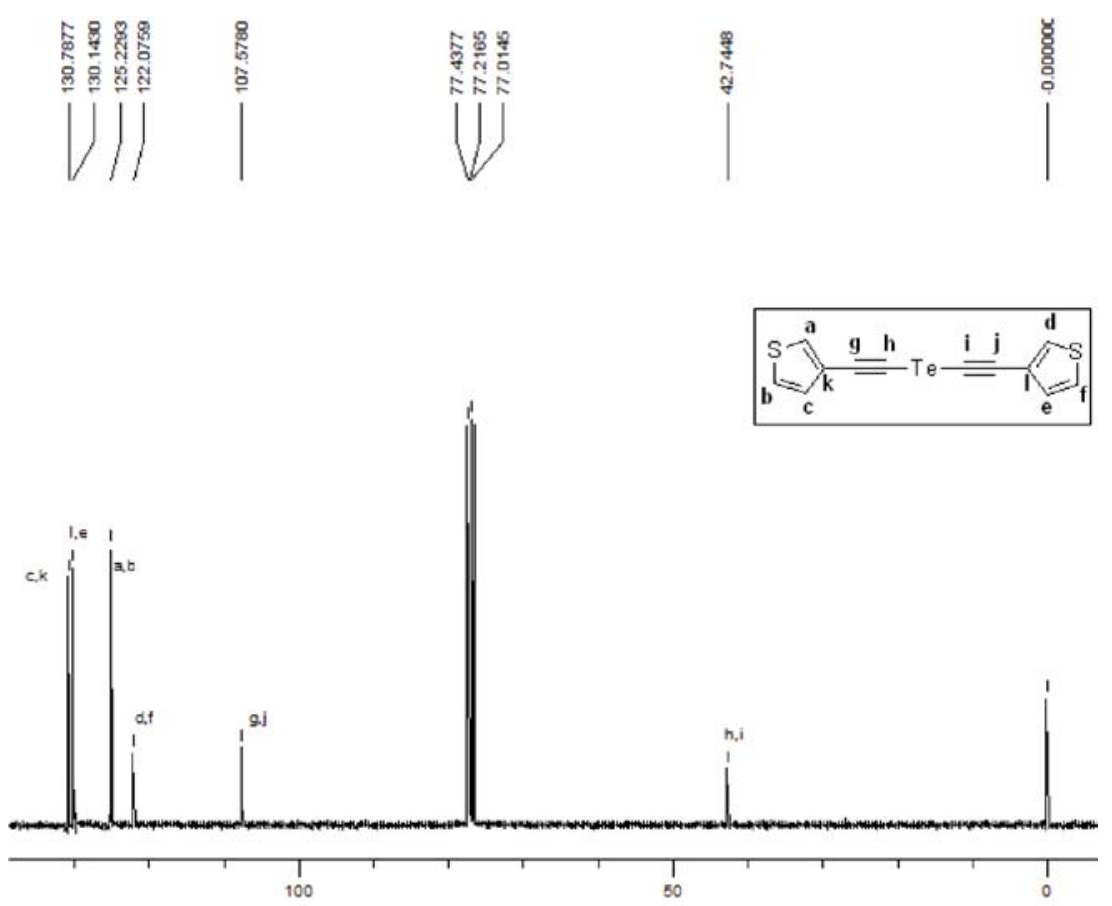

Figure S12. ${ }^{13} \mathrm{C}$ NMR $\left(\mathrm{CDCl}_{3}, 300 \mathrm{MHz}\right)$ of $\mathbf{1 f}$. 

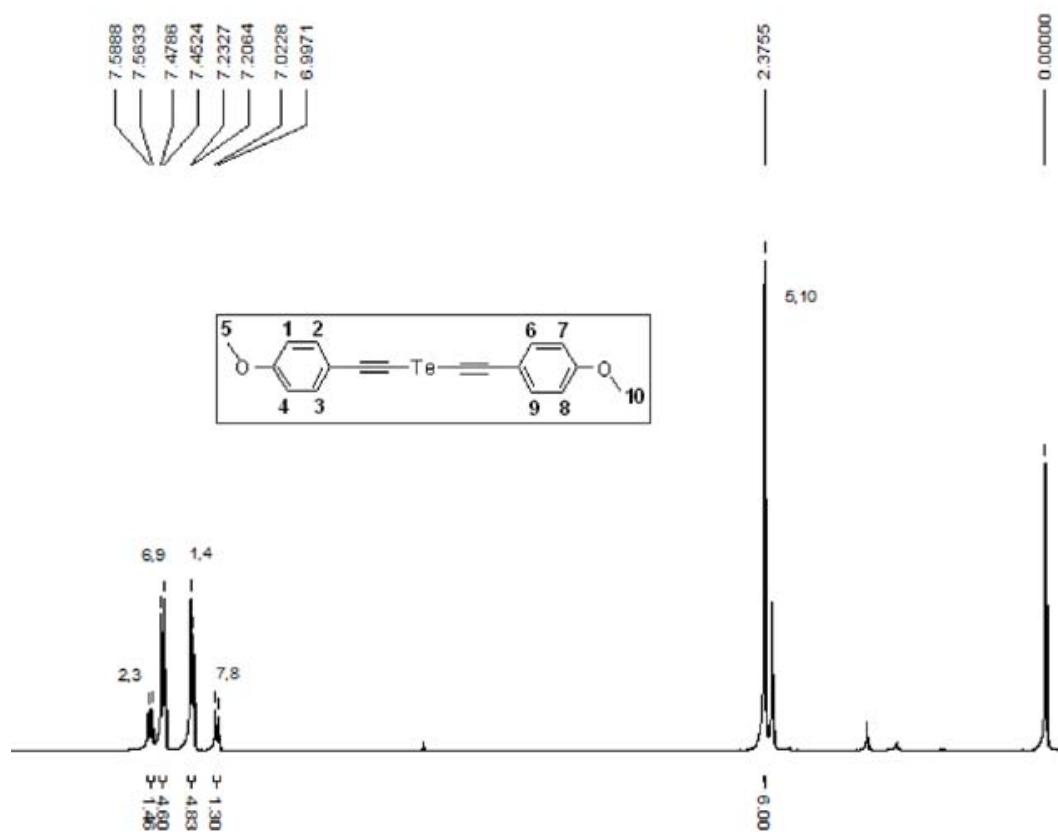

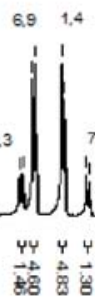

Figure S13. ${ }^{1} \mathrm{H} \mathrm{NMR}\left(\mathrm{CDCl}_{3}, 300 \mathrm{MHz}\right)$ of $\mathbf{1 g}$.
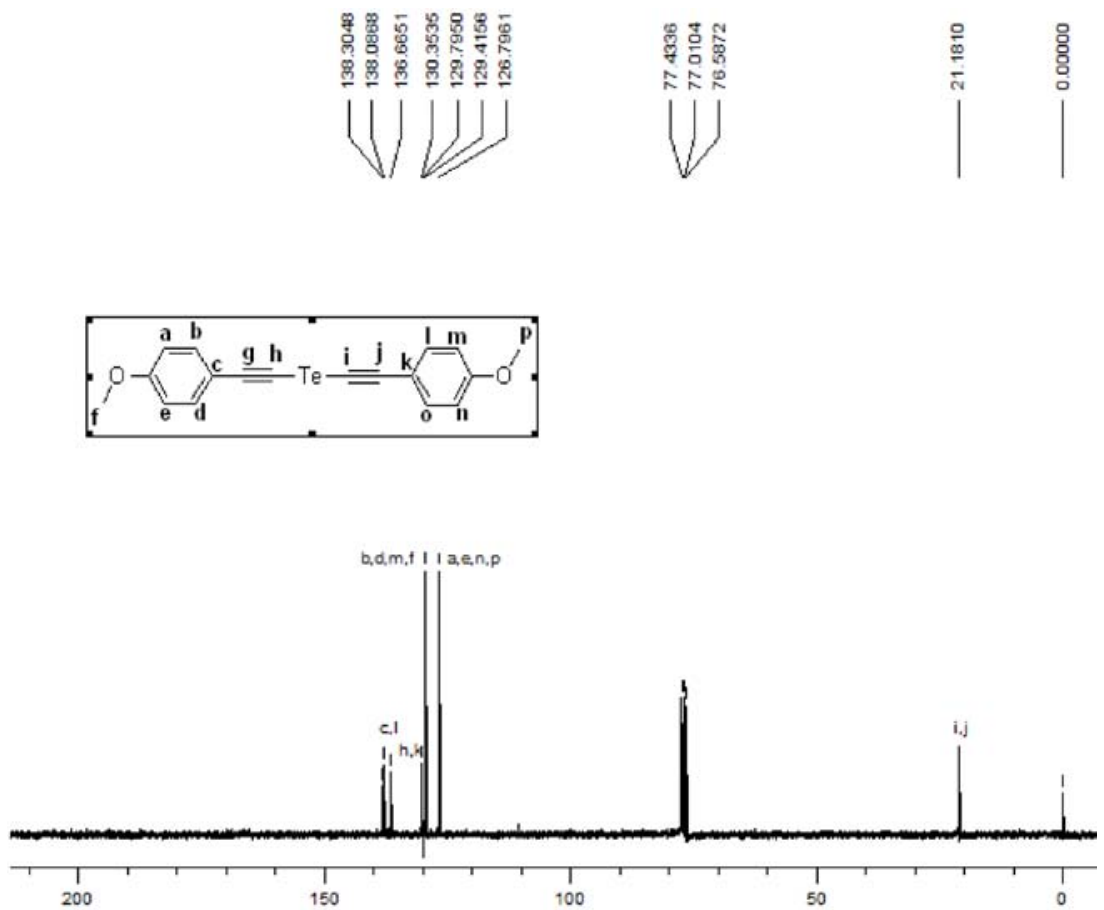

Figure S14. ${ }^{13} \mathrm{C}$ NMR $\left(\mathrm{CDCl}_{3}, 300 \mathrm{MHz}\right)$ of $\mathbf{1 g}$. 

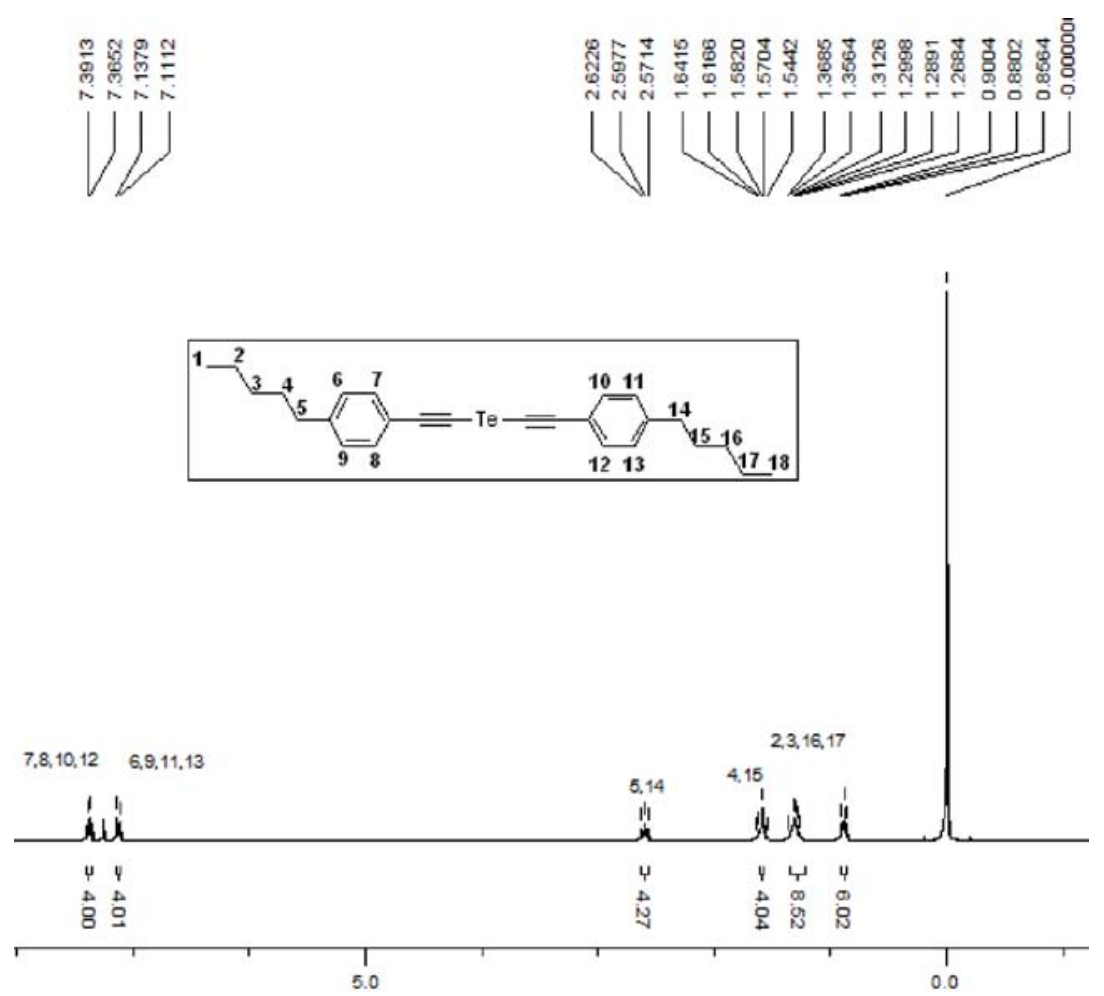

Figure S15. ${ }^{1} \mathrm{H} \mathrm{NMR}\left(\mathrm{CDCl}_{3}, 300 \mathrm{MHz}\right)$ of $\mathbf{1 h}$.
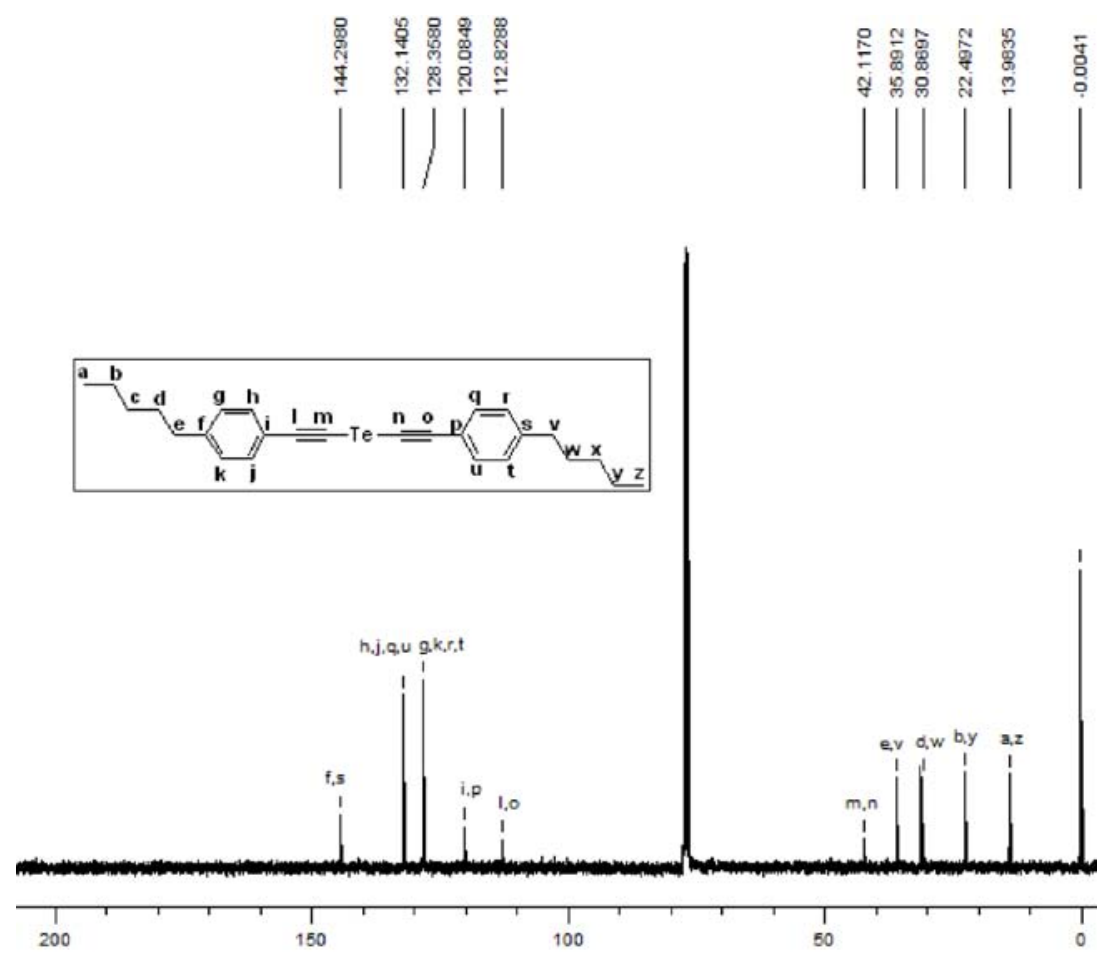

Figure S16. ${ }^{13} \mathrm{C}$ NMR $\left(\mathrm{CDCl}_{3}, 300 \mathrm{MHz}\right)$ of $\mathbf{1 h}$. 

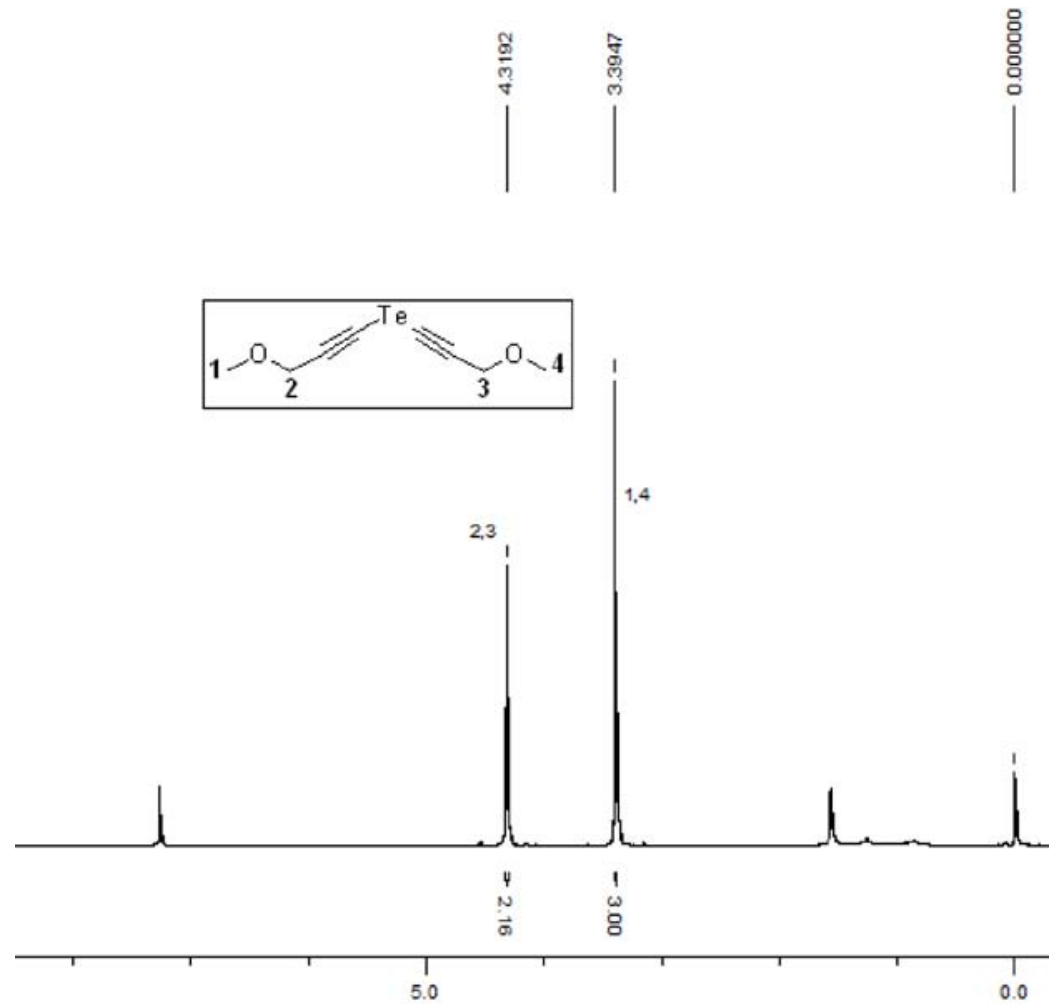

Figure S17. ${ }^{1} \mathrm{H} \mathrm{NMR}\left(\mathrm{CDCl}_{3}, 300 \mathrm{MHz}\right)$ of $\mathbf{1 i}$.
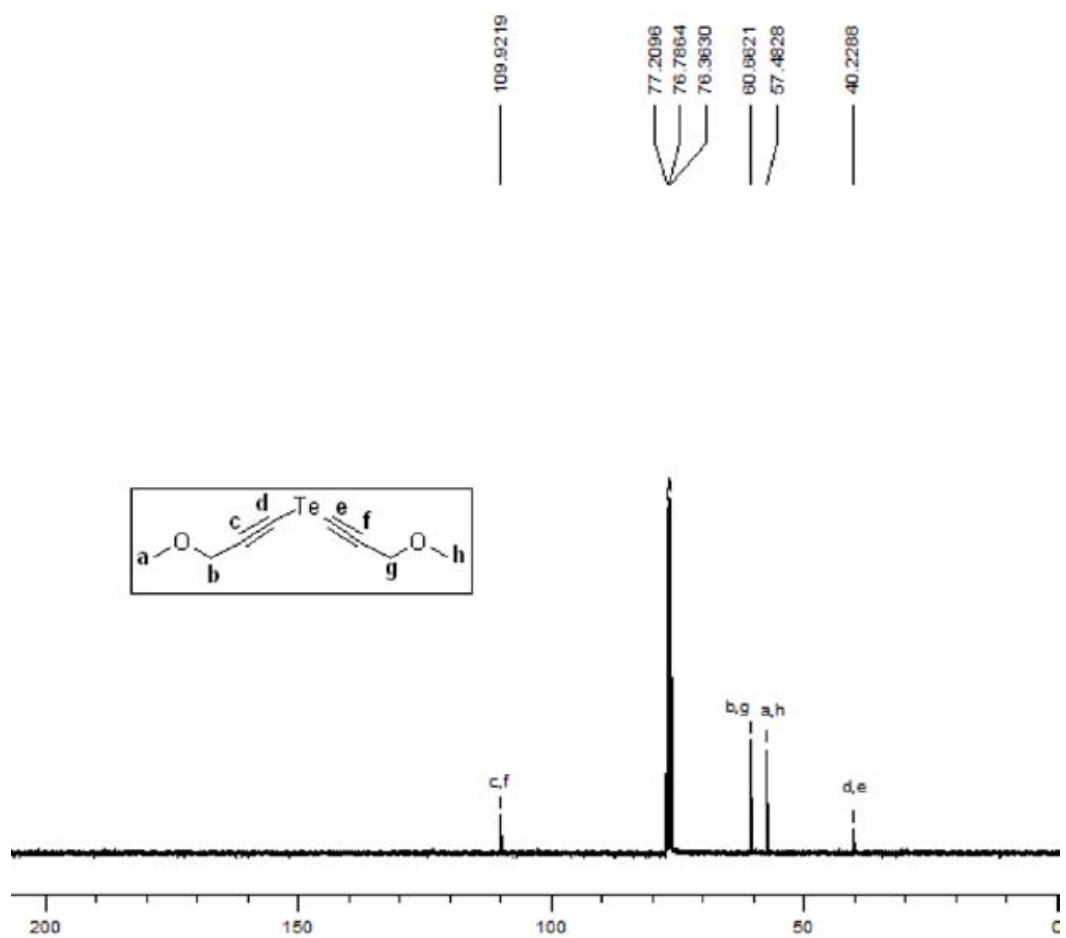

Figure S18. ${ }^{13} \mathrm{C}$ NMR $\left(\mathrm{CDCl}_{3}, 300 \mathrm{MHz}\right)$ of $\mathbf{1 i}$. 

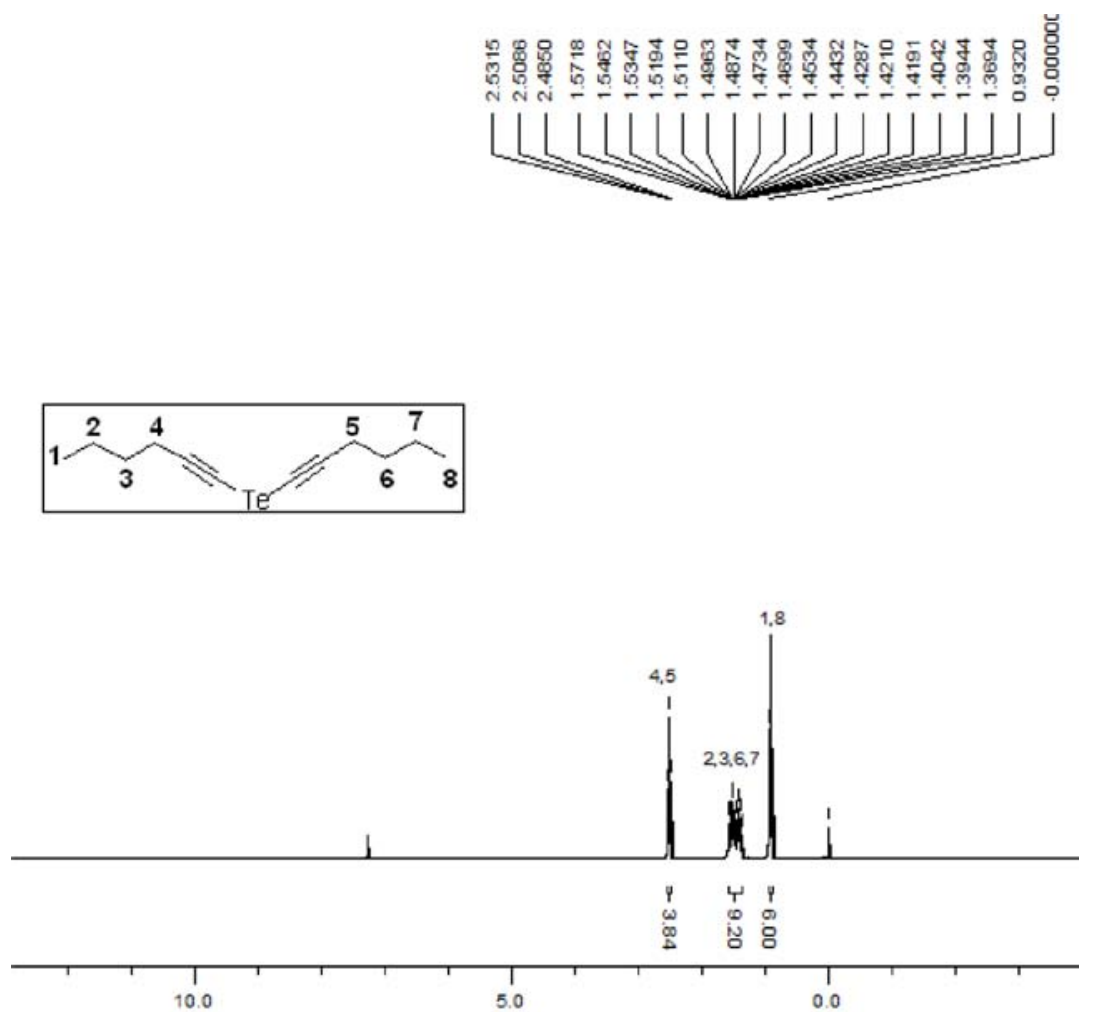

Figure S19. ${ }^{1} \mathrm{H} \mathrm{NMR}\left(\mathrm{CDCl}_{3}, 300 \mathrm{MHz}\right)$ of $\mathbf{1 j}$.
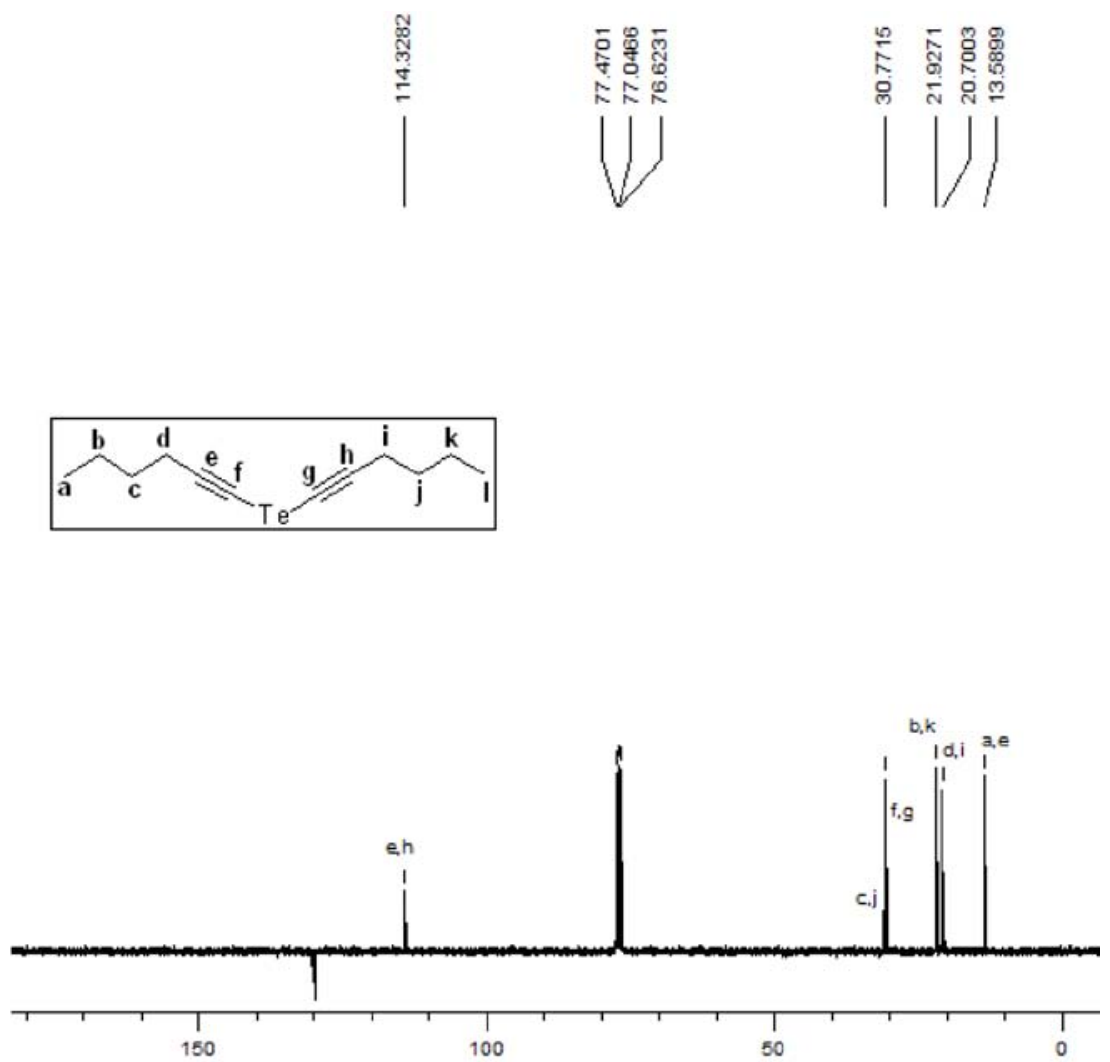

Figure S20. ${ }^{13} \mathrm{C}$ NMR $\left(\mathrm{CDCl}_{3}, 300 \mathrm{MHz}\right)$ of $\mathbf{1 j}$. 

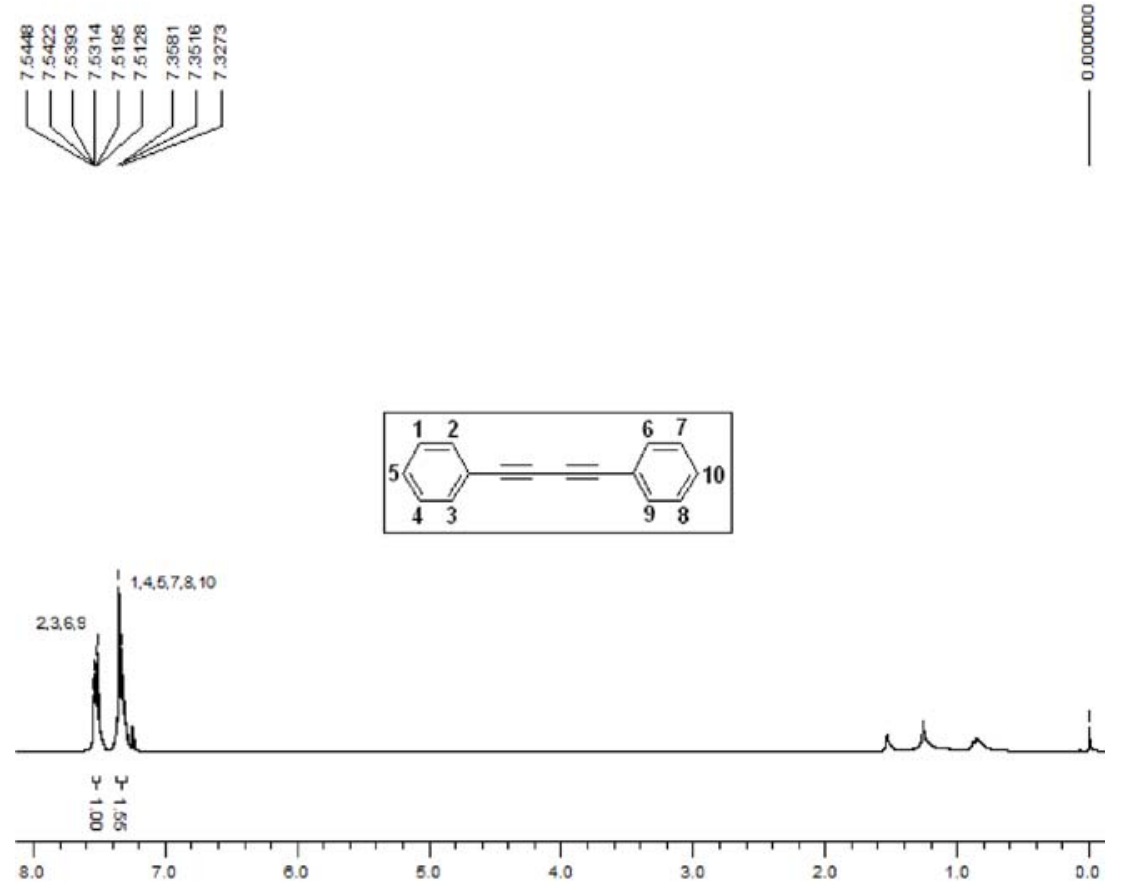

Figure S21. ${ }^{1} \mathrm{H} \mathrm{NMR}\left(\mathrm{CDCl}_{3,}, 300 \mathrm{MHz}\right)$ of $\mathbf{2 a}$.
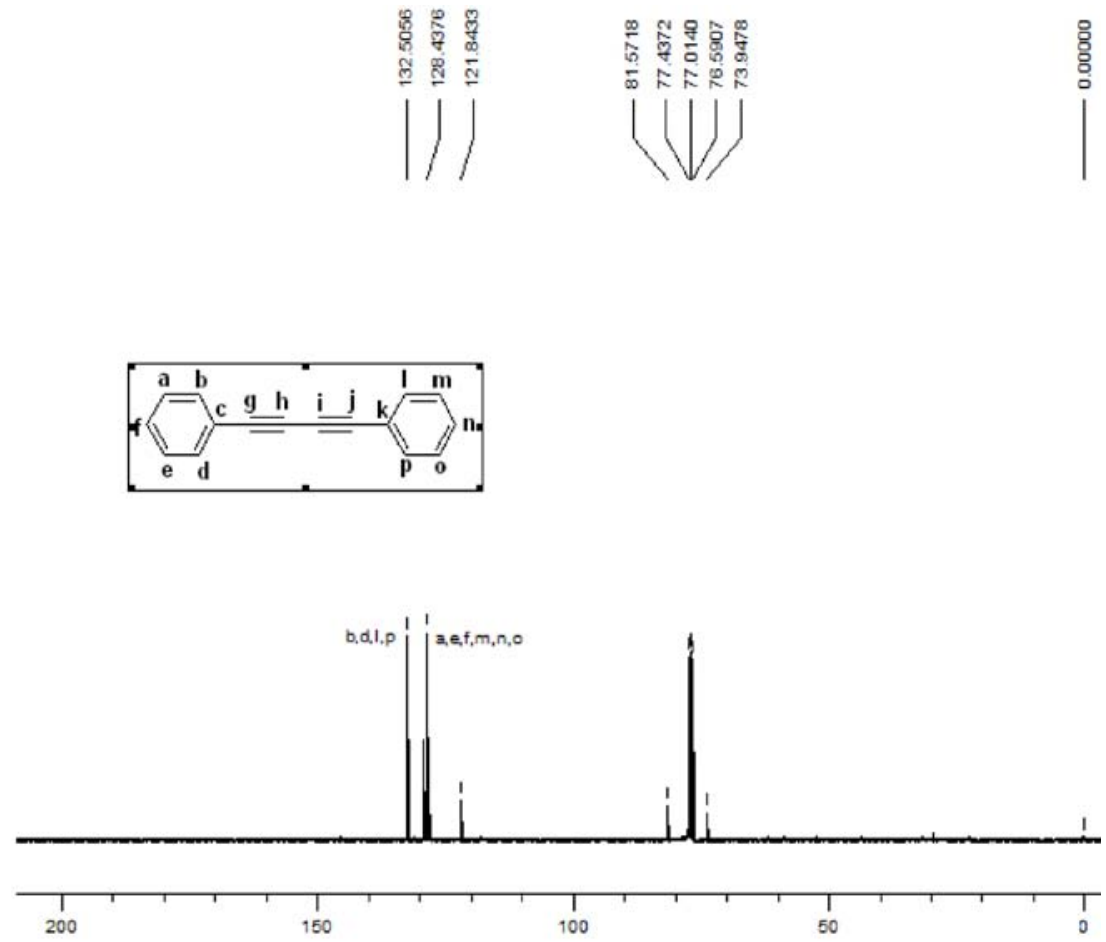

Figure S22. ${ }^{13} \mathrm{C}$ NMR $\left(\mathrm{CDCl}_{3}, 300 \mathrm{MHz}\right)$ of $\mathbf{2 a}$. 

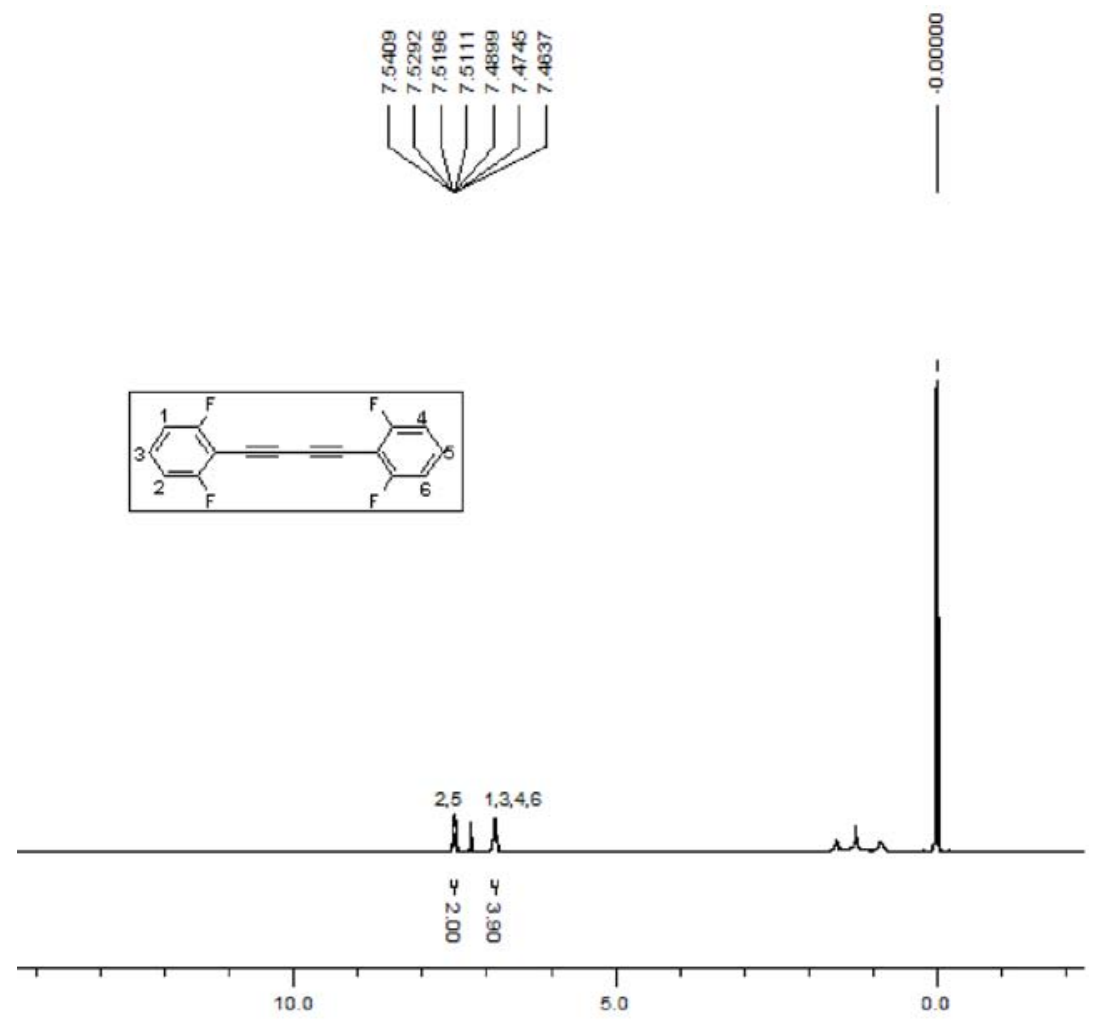

Figure S23. ${ }^{1} \mathrm{H} \mathrm{NMR}\left(\mathrm{CDCl}_{3,}, 300 \mathrm{MHz}\right)$ of $\mathbf{2 b}$.
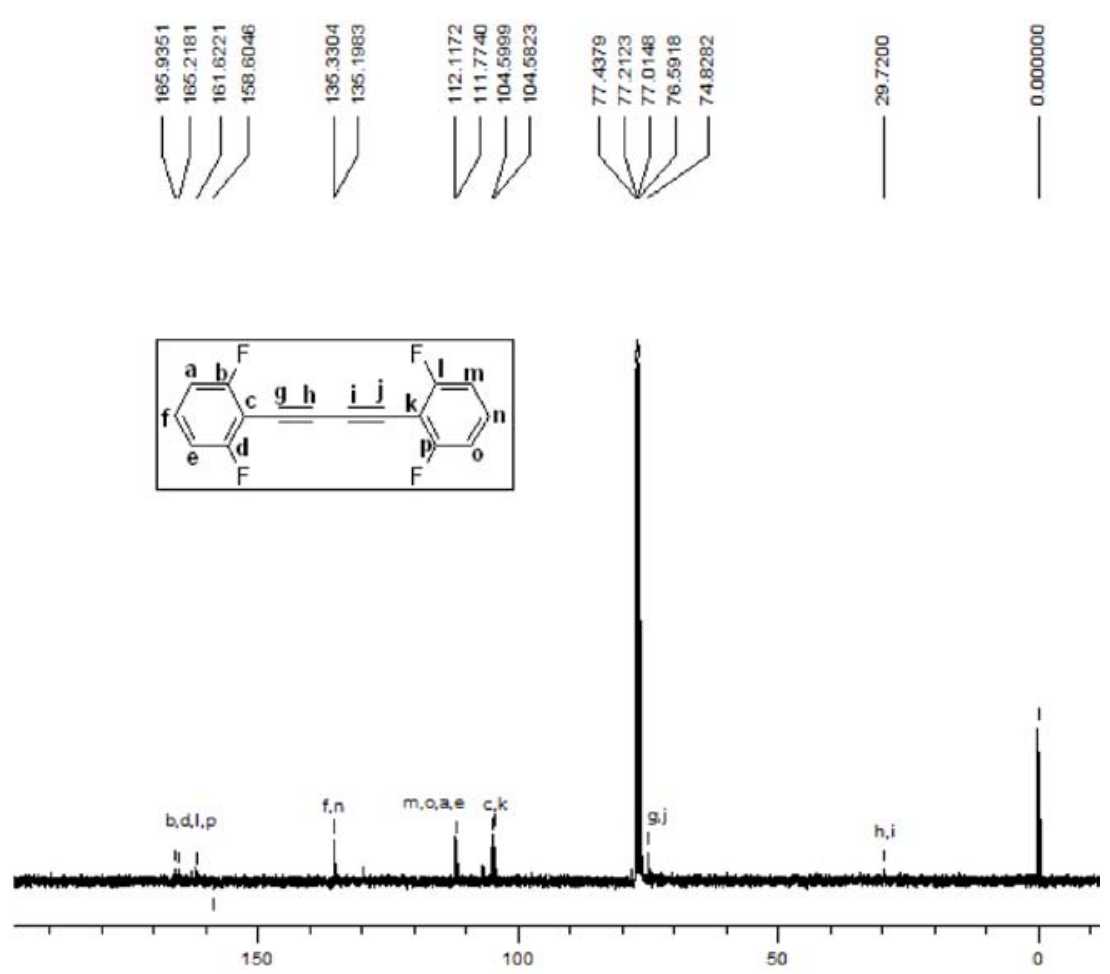

Figure S24. ${ }^{13} \mathrm{C}$ NMR $\left(\mathrm{CDCl}_{3}, 300 \mathrm{MHz}\right)$ of $\mathbf{2 b}$. 

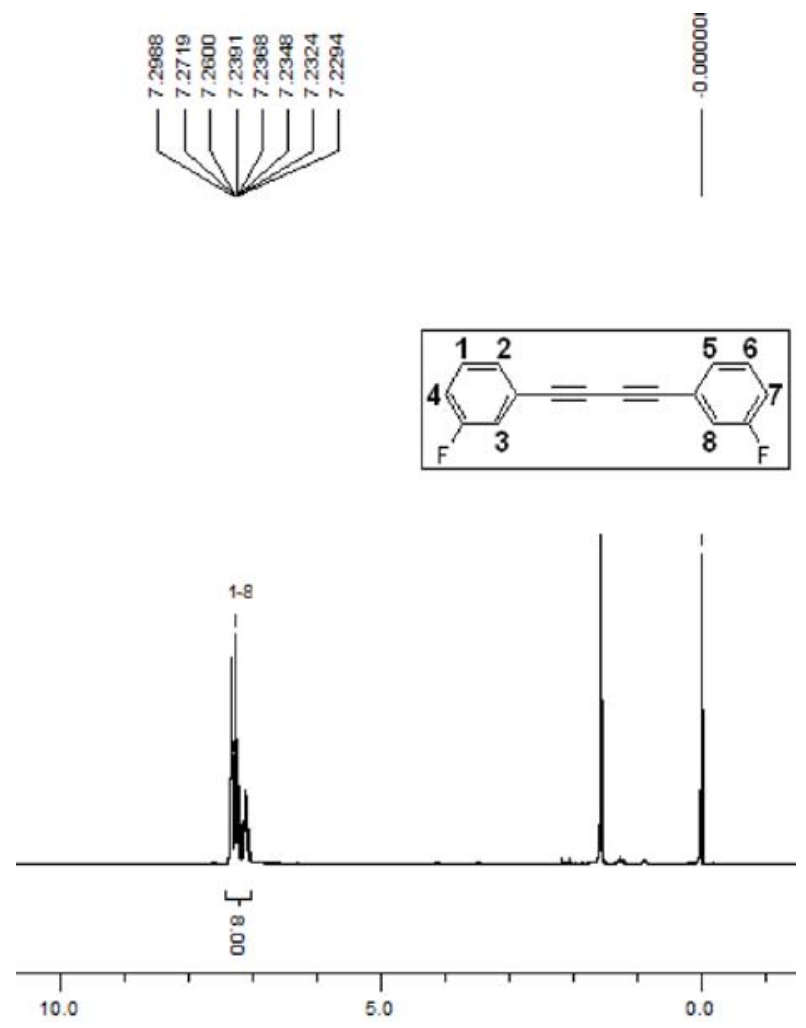

Figure S25. ${ }^{1} \mathrm{H} \mathrm{NMR}\left(\mathrm{CDCl}_{3}, 300 \mathrm{MHz}\right)$ of $\mathbf{2 c}$.
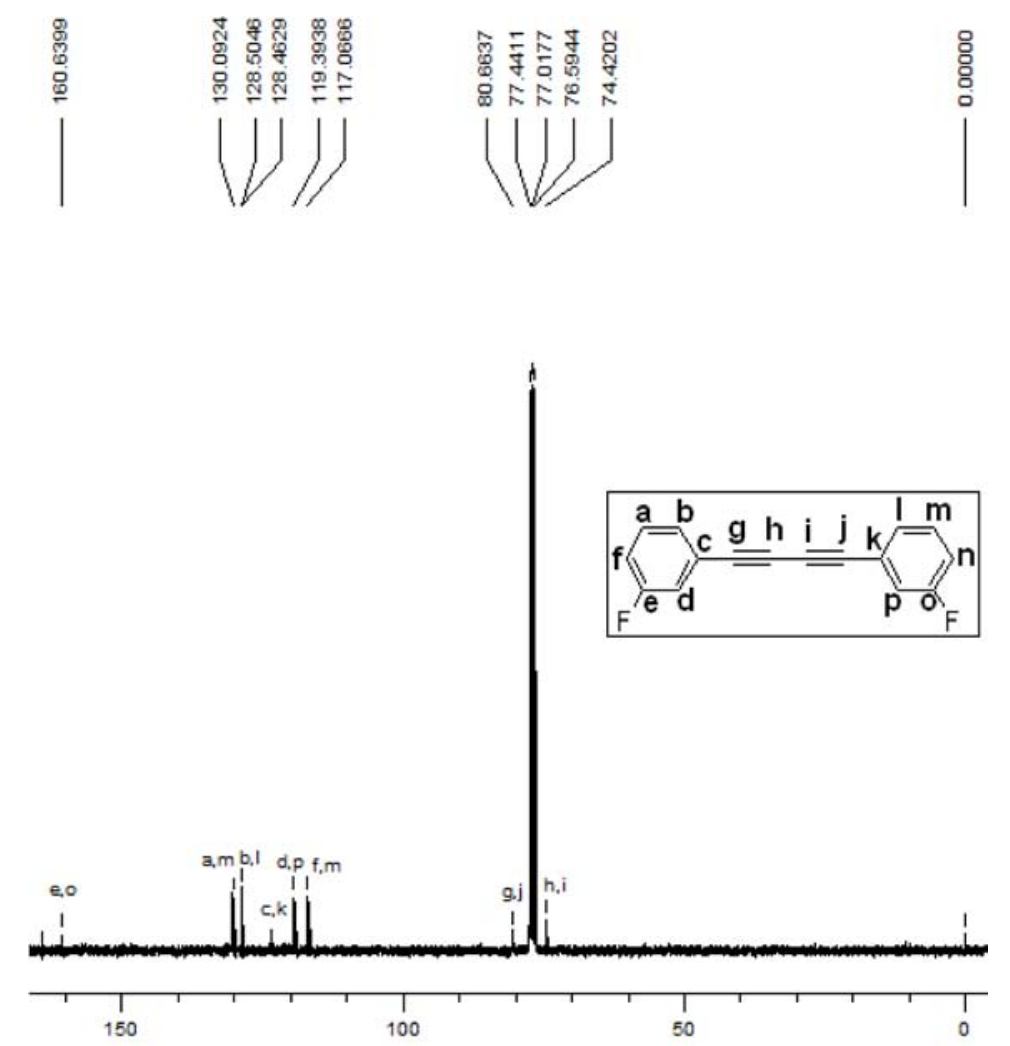

Figure S26. ${ }^{13} \mathrm{C}$ NMR $\left(\mathrm{CDCl}_{3}, 300 \mathrm{MHz}\right)$ of $\mathbf{2 c}$. 


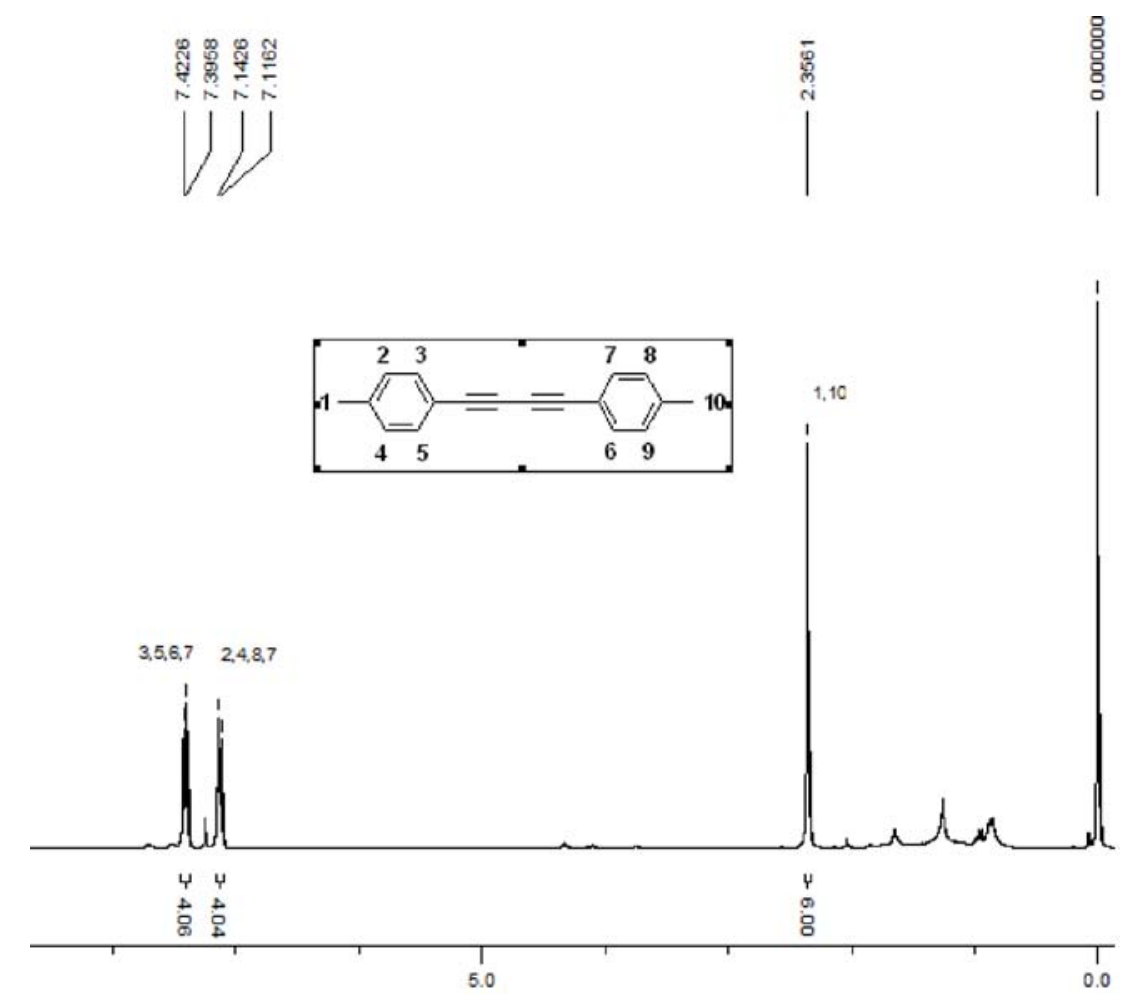

Figure S27. ${ }^{1} \mathrm{H} \mathrm{NMR}\left(\mathrm{CDCl}_{3}, 300 \mathrm{MHz}\right)$ of $\mathbf{2 d}$.

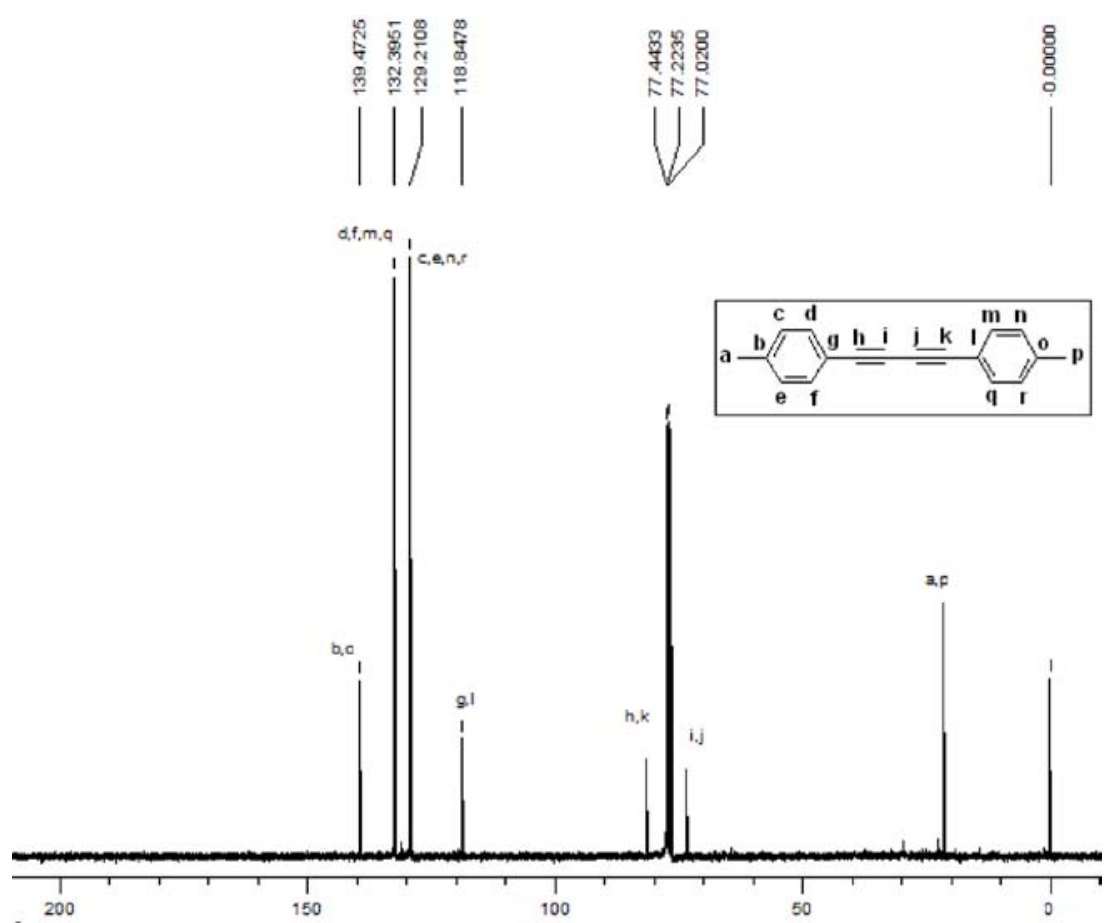

Figure S28. ${ }^{13} \mathrm{C} \mathrm{NMR}\left(\mathrm{CDCl}_{3}, 300 \mathrm{MHz}\right)$ of $\mathbf{2 d}$. 

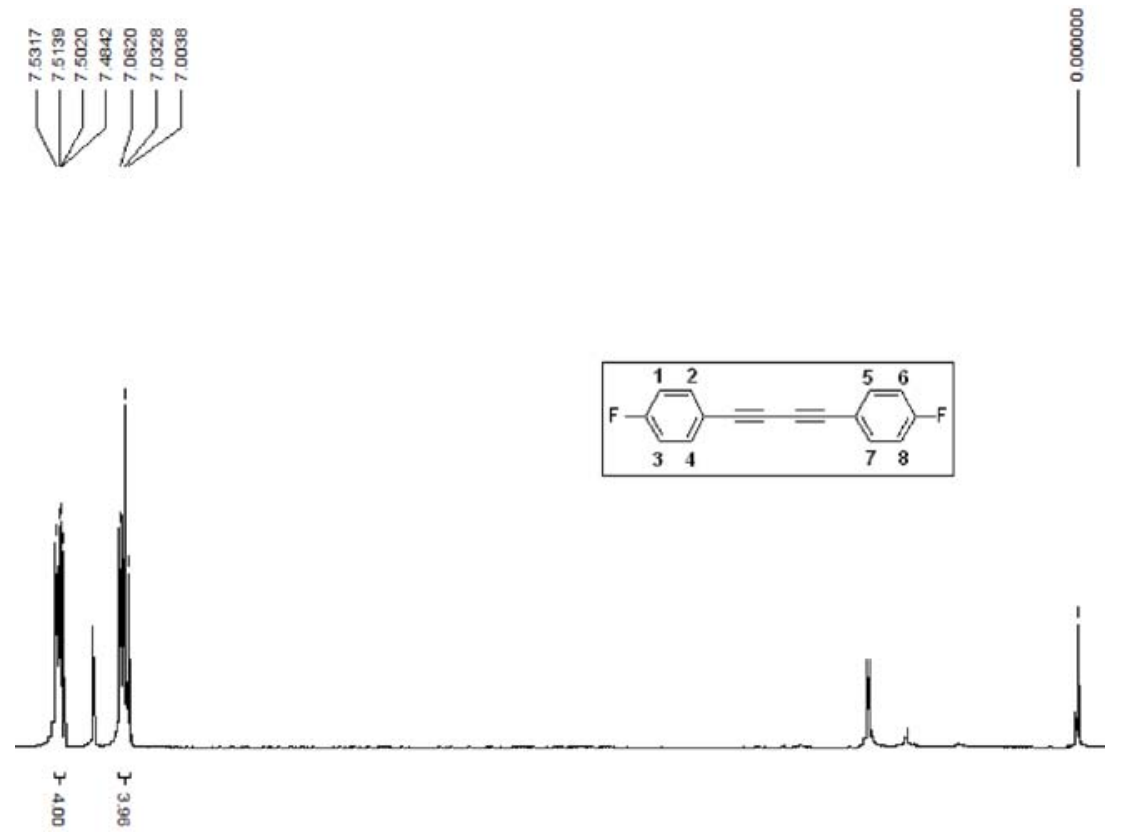

Figure S29. ${ }^{1} \mathrm{H} \mathrm{NMR}\left(\mathrm{CDCl}_{3,}, 300 \mathrm{MHz}\right)$ of $\mathbf{2 e}$.
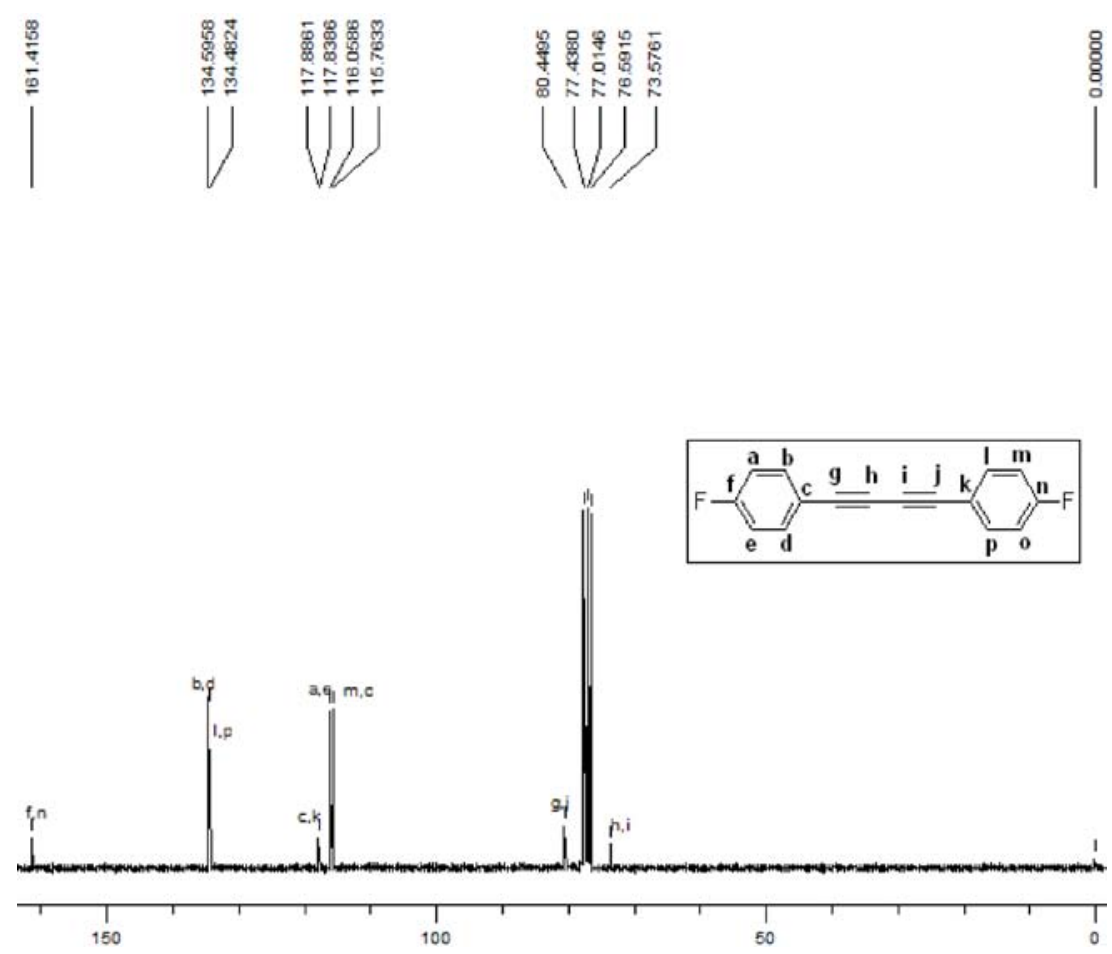

Figure S30. ${ }^{13} \mathrm{C}$ NMR $\left(\mathrm{CDCl}_{3} 300 \mathrm{MHz}\right)$ of $\mathbf{2 e}$. 

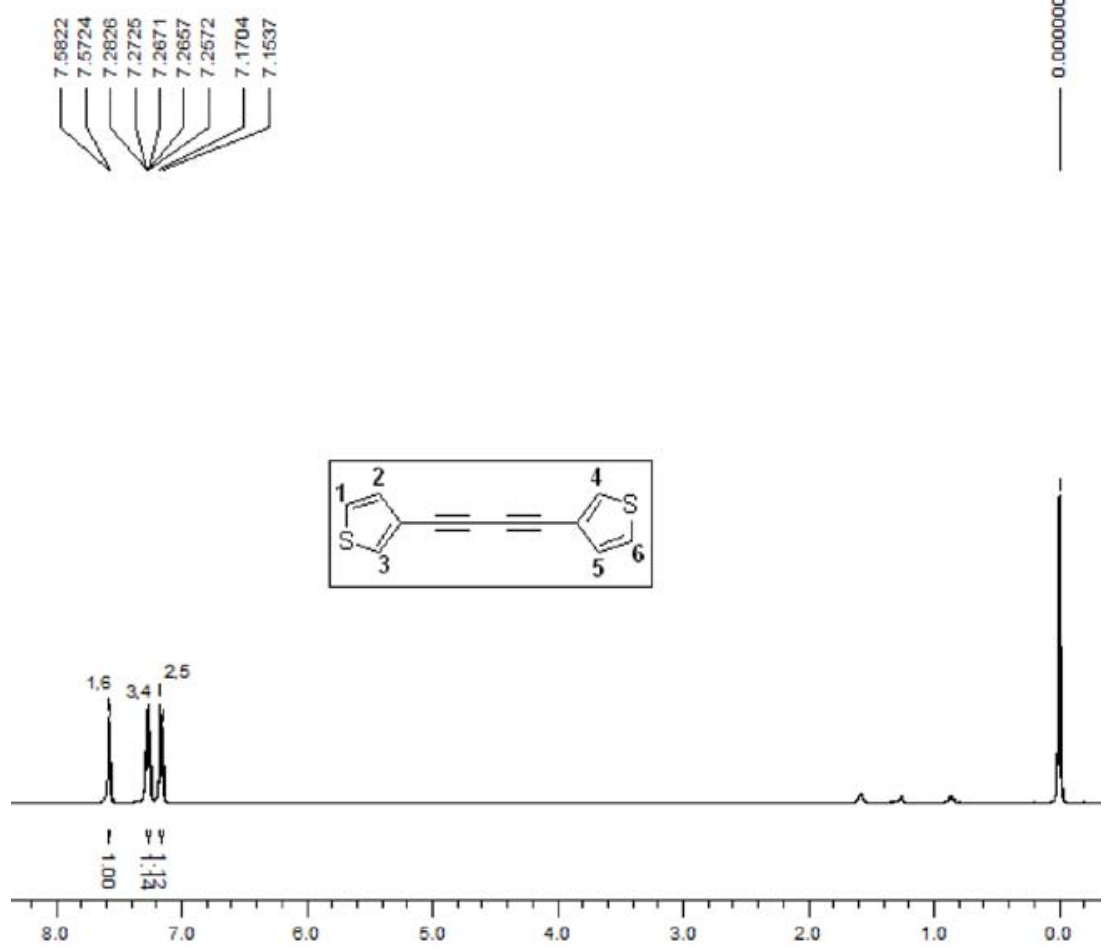

Figure S31. ${ }^{1} \mathrm{H} \mathrm{NMR}\left(\mathrm{CDCl}_{3}, 300 \mathrm{MHz}\right)$ of $\mathbf{2 f}$.
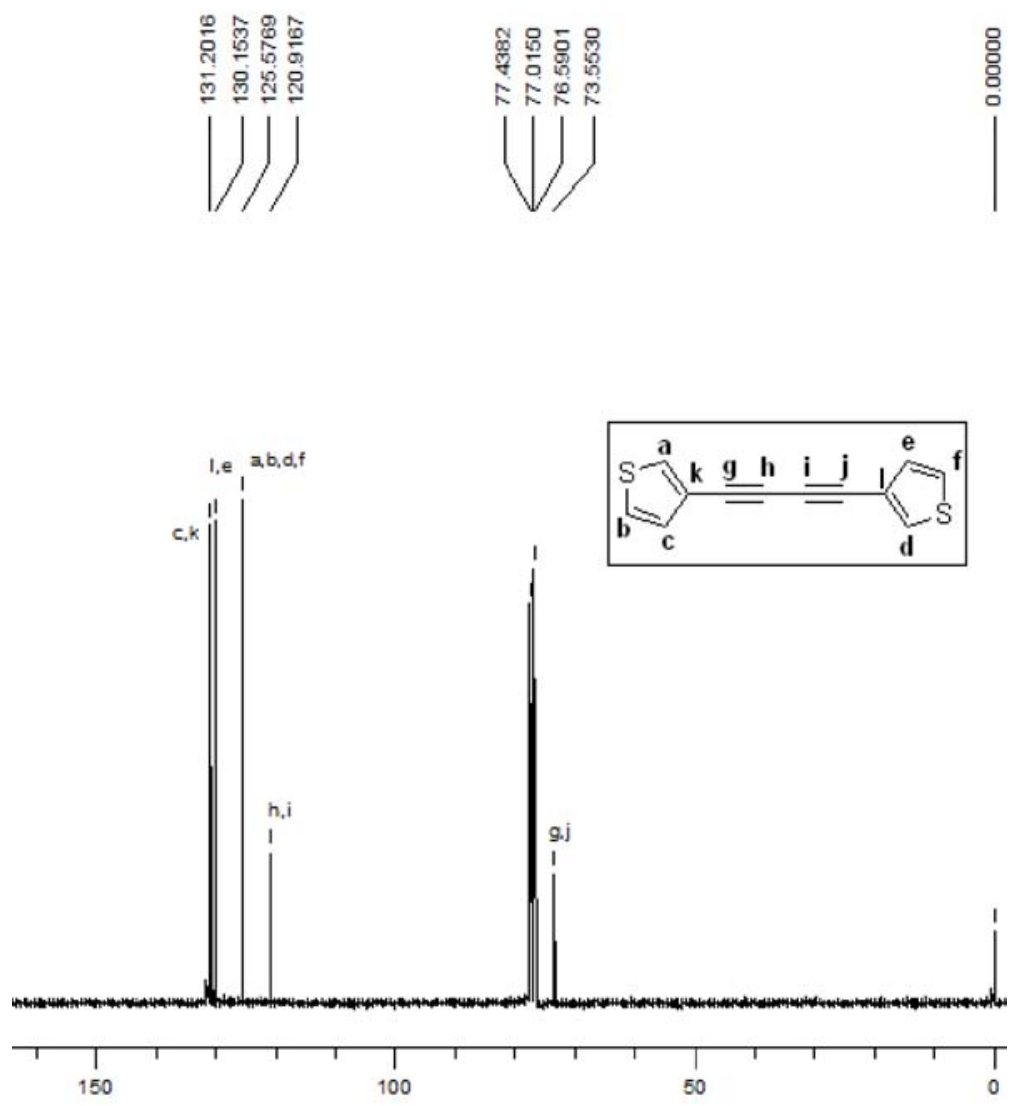

Figure S32. ${ }^{13} \mathrm{C}$ NMR $\left(\mathrm{CDCl}_{3}, 300 \mathrm{MHz}\right)$ of $\mathbf{2 f}$. 

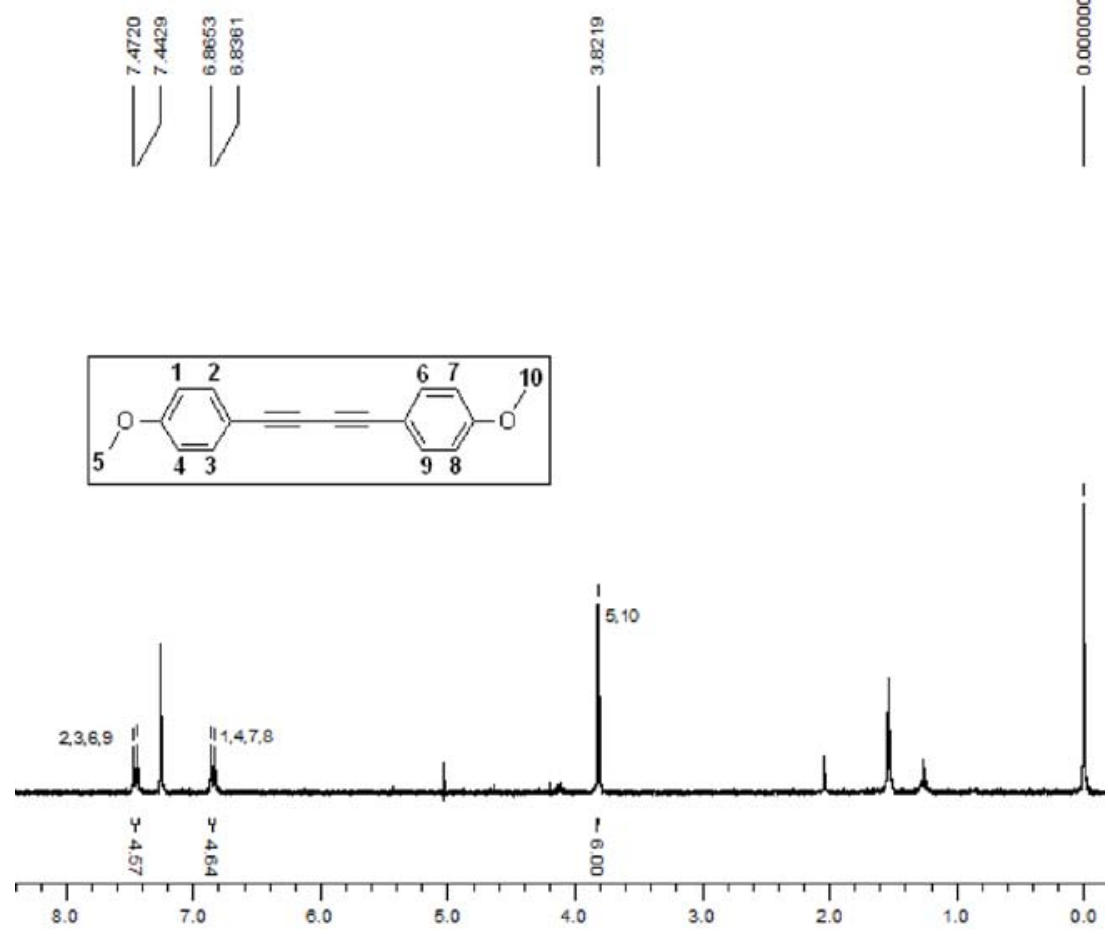

Figure S33. ${ }^{1} \mathrm{H}$ NMR $\left(\mathrm{CDCl}_{3}, 300 \mathrm{MHz}\right)$ of $\mathbf{2 g}$.

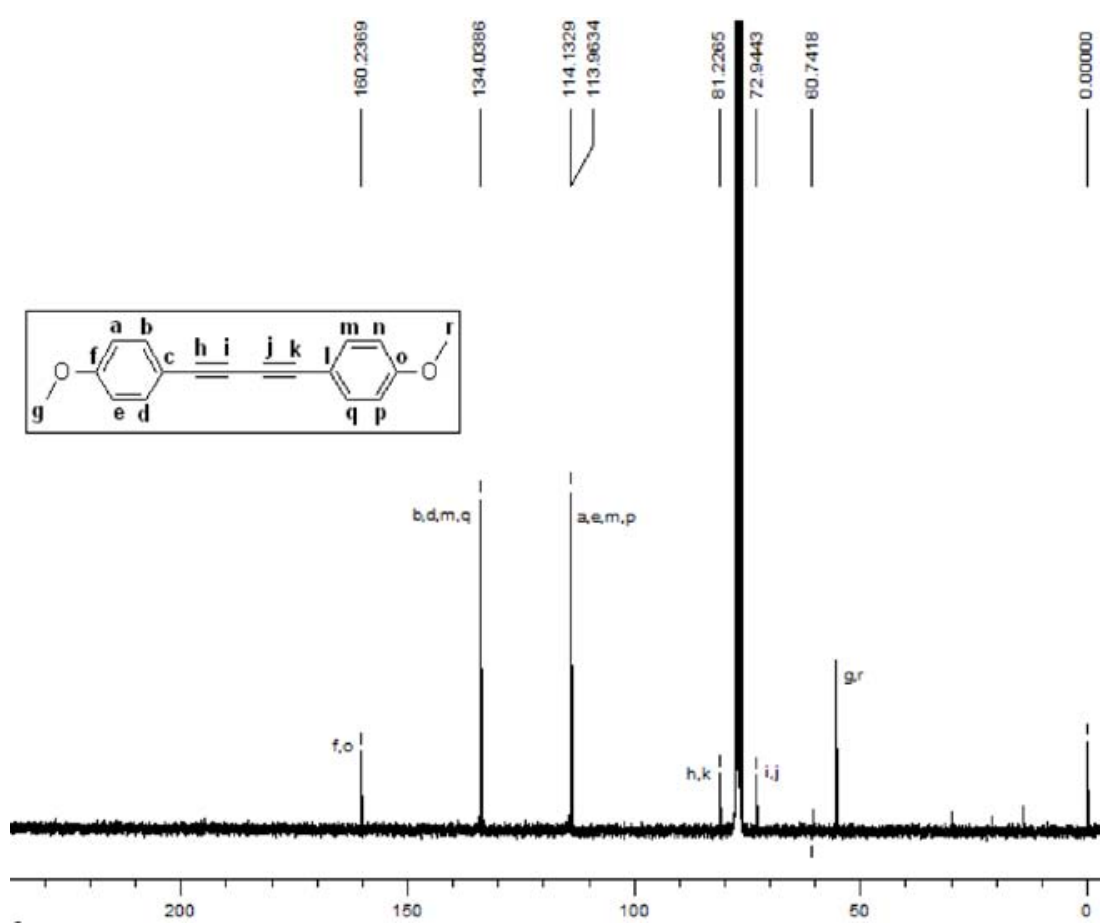

Figure S34. ${ }^{13} \mathrm{C}$ NMR $\left(\mathrm{CDCl}_{3}, 300 \mathrm{MHz}\right)$ of $\mathbf{2 g}$. 

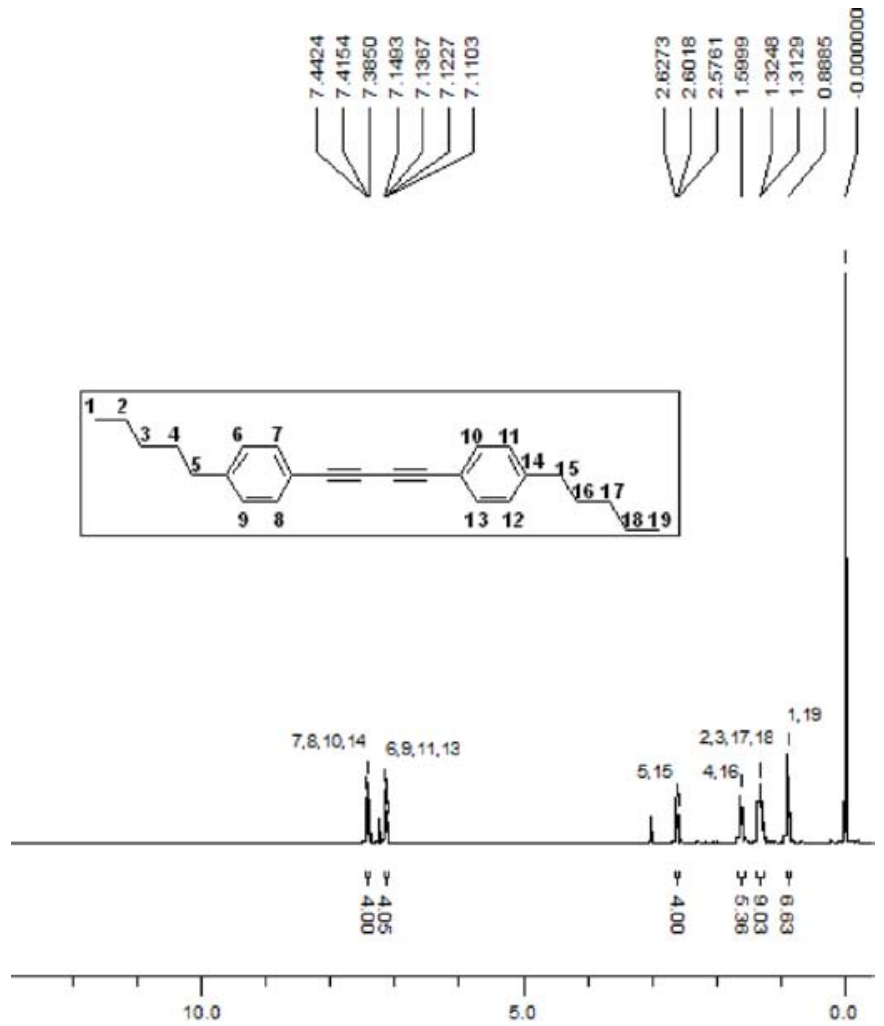

Figure S35. ${ }^{1} \mathrm{H} \mathrm{NMR}\left(\mathrm{CDCl}_{3}, 300 \mathrm{MHz}\right)$ of $\mathbf{2 h}$.
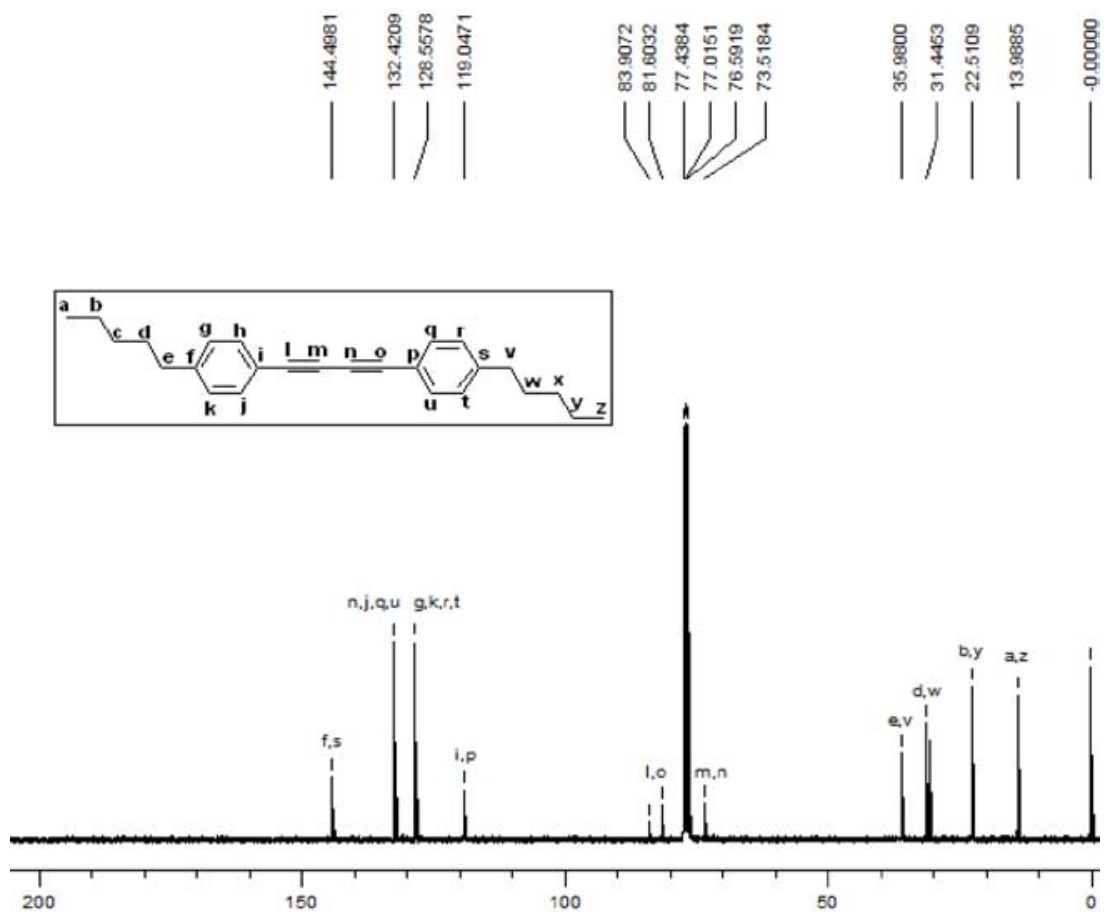

Figure S36. ${ }^{13} \mathrm{C}$ NMR $\left(\mathrm{CDCl}_{3}, 300 \mathrm{MHz}\right)$ of $\mathbf{2 h}$. 


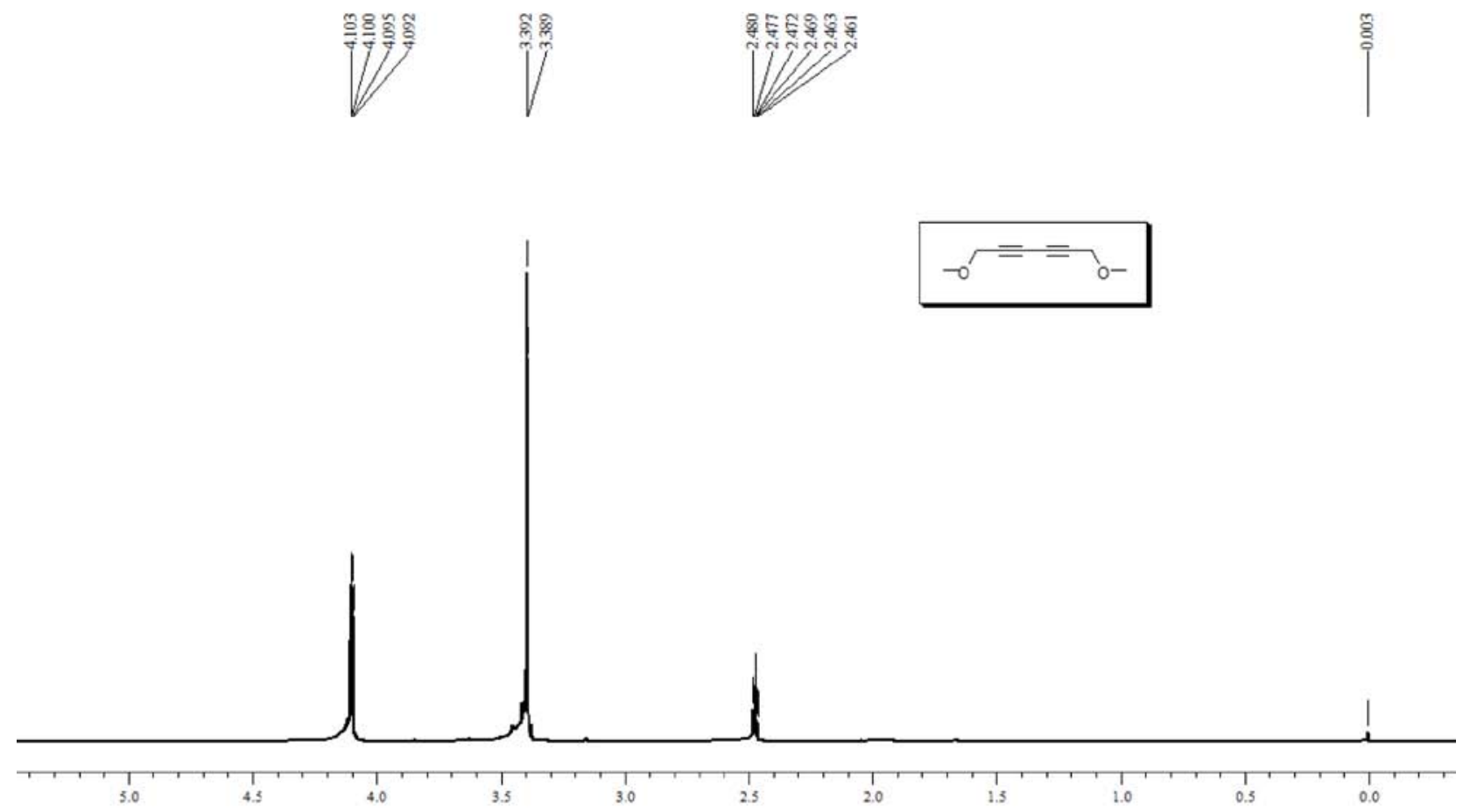

Figure S37. ${ }^{1} \mathrm{H} \mathrm{NMR}\left(\mathrm{CDCl}_{3}, 300 \mathrm{MHz}\right)$ of $\mathbf{2 i}$.
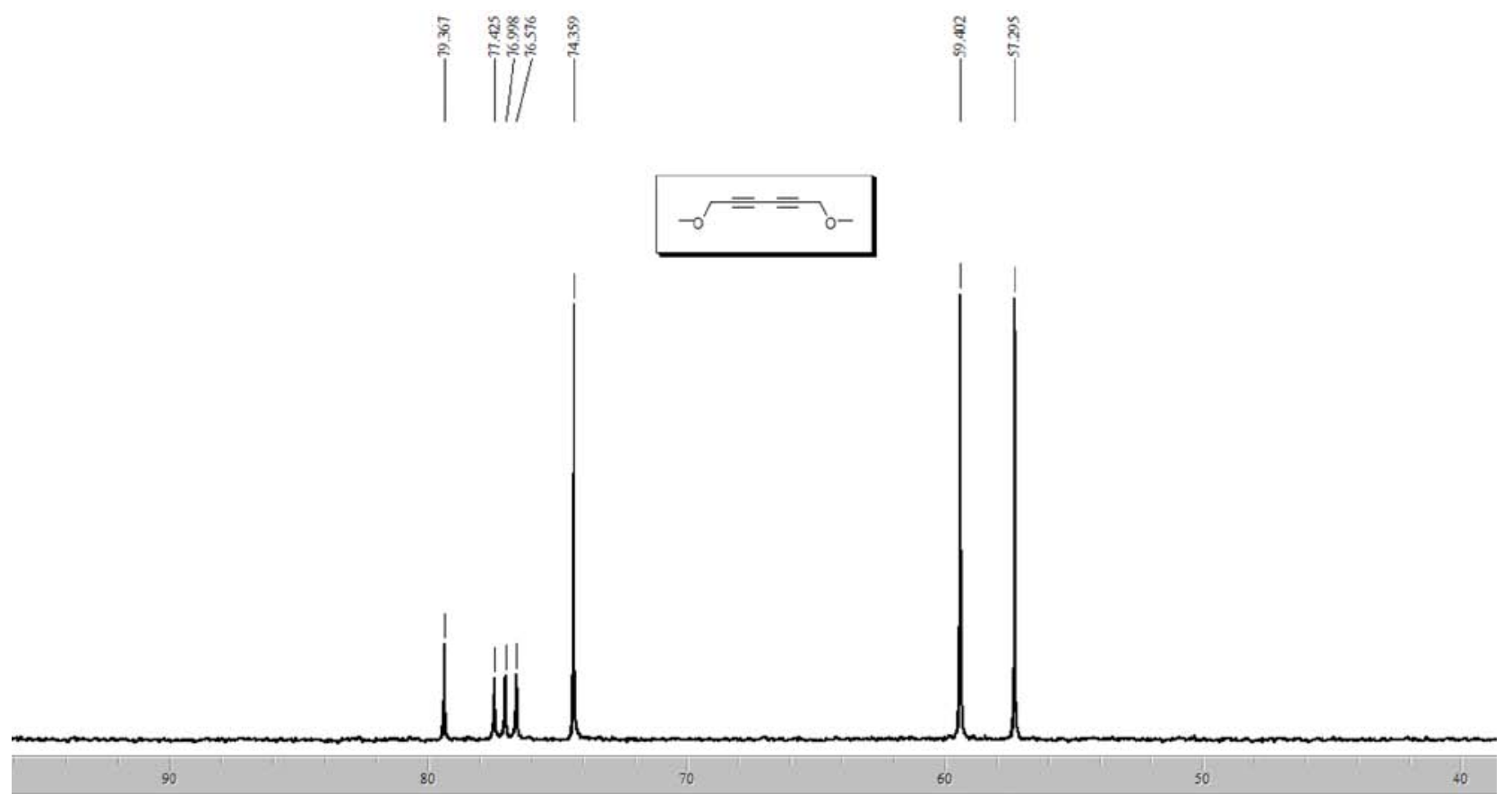

Figure S38. ${ }^{13} \mathrm{C}$ NMR $\left(\mathrm{CDCl}_{3}, 300 \mathrm{MHz}\right)$ of $\mathbf{2 i}$. 